Preprint typeset in JHEP style - HYPER VERSION

FSU-HEP-060426, UH-511-1087-06

\title{
Collider and Dark Matter Searches in Models with Mixed Modulus-Anomaly Mediated SUSY Breaking
}

\author{
Howard Baer $^{a}$, Eun-Kyung Park ${ }^{a}$, Xerxes Tata ${ }^{b}$ and Ting T. Wang ${ }^{a}$ \\ ${ }^{a}$ Department of Physics, Florida State University Tallahassee, FL 32306, USA \\ ${ }^{b}$ Department of Physics and Astronomy, University of Hawaii, Honolulu, HI 96822, USA \\ E-mail: baer@hep.fsu.edu, epark@hep.fsu.edu, \\ tata@phys.hawaii.edu,tingwang@hep.fsu.edu
}

\begin{abstract}
We investigate the phenomenology of supersymmetric models where moduli fields and the Weyl anomaly make comparable contributions to SUSY breaking effects in the observable sector of fields. This mixed modulus-anomaly mediated supersymmetry breaking (MM-AMSB) scenario is inspired by models of string compactification with fluxes, which have been shown to yield a de Sitter vacuum (as in the recent construction by Kachru et al). The phenomenology depends on the so-called modular weights which, in turn, depend on the location of various fields in the extra dimensions. We find that the model with zero modular weights gives mass spectra characterized by very light top squarks and/or tau sleptons, or where $M_{1} \sim-M_{2}$ so that the bino and wino are approximately degenerate. The top squark mass can be in the range required by successful electroweak baryogenesis. The measured relic density of cold dark matter can be obtained via top squark co-annihilation at low $\tan \beta$, tau slepton co-annihilation at large $\tan \beta$ or via binowino coannihilation. Then, we typically find low rates for direct and indirect detection of neutralino dark matter. However, essentially all the WMAP-allowed parameter space can be probed by experiments at the CERN LHC, while significant portions may also be explored at an $e^{+} e^{-}$collider with $\sqrt{s}=0.5-1 \mathrm{TeV}$. We also investigate a case with non-zero modular weights. In this case, co-annihilation, $A$-funnel annihilation and bulk annihilation of neutralinos are all allowed. Results for future colliders are qualitatively similar, but prospects for indirect dark matter searches via gamma rays and anti-particles are somewhat better.
\end{abstract}

Keywords: Supersymmetry Phenomenology, Supersymmetric Standard Model, Dark Matter. 


\section{Introduction}

One of the main goals of string phenomenology is to connect string theory to observable phenomena at colliding beam, or other, experiments. This difficult enterprise may be made tractable by merging of top-down and bottom-up approaches of connecting weak scale phenomena to superstring theory valid at and above the string scale. The many theoretical and phenomenological advantages of weak scale supersymmetry provide a target for what string theory must yield at energy scales of order $\sim 1 \mathrm{TeV}$. Alternatively, the discovery of weak scale supersymmetry and tabulation of the superparticle properties could shed important light on the nature of physics at the string scale.

A significant hurdle to the implementation of the string phenomenology program is the existence of many flat directions in the space of scalar fields (the moduli), since many physically relevant couplings are determined by the ground state values of these moduli. Determining these requires that the flat directions be lifted and stabilized, at least in a controlled approximation, so that the ground state whose properties determine low energy physics can be reliably determined. This has been facilitated by a new class of compactifications, where the extra spatial dimensions are curled up to small sizes with fluxes of additional fields turned on along these extra dimensions. The presence of these fluxes leads to calculable minima in the potential of the moduli, and represents a starting point of the program for discovering a string ground state that will lead to the (supersymmetric) Standard Model at low energies, and which is consistent with constraints from cosmology. By the latter, we refer to upper bounds on the energy density of moduli from the age of the universe, to constraints on their decays after nucleosynthesis, and to the observed acceleration of supernovae at high red shifts which implies a de Sitter universe.

Recently, Kachru, Kallosh, Linde and Trivedi (KKLT) [1 provided a concrete model, based on type-IIB superstrings, of compactification with fluxes to a Calabi-Yau orientifold. The background fluxes (non-zero vacuum expectation values of various field strengths) allow one to stabilize the complex structure moduli (that determine the shape of the compactified manifold) and the dilaton field, and remove these fields from the low energy theory because they are heavy. The remaining size modulus is stabilized by a non-perturbative mechanism such as gaugino condensation on a $D 7$ brane. At this point, the vacuum of the theory is of anti-de Sitter (AdS) type, in contradiction to cosmological observations. The addition of a non-supersymmetric anti-brane $(\overline{D 3})$ breaks the $N=1$ supersymmetry and lifts the vacuum minimum to zero or positive values, yielding a de Sitter universe as required by cosmological measurements referred to above. Due to the warping induced by the fluxes, the addition of the $\overline{D 3}$ brane also breaks supersymmetry by a very small amount. The resulting low energy theory thus has no unwanted light moduli, has a broken supersymmetry, and a positive cosmological constant. There is, however, still a need for a concrete implementation of the KKLT idea with an explicit Calabi-Yau space and choice of fluxes that yields a ground state leading to the observed low energy phenomenology.

Recently, several papers have analyzed the structure of the ensuing soft supersymmetry breaking terms (SSB) in models based on the KKLT proposal[2]. A very interesting result that they find is that these terms can receive comparable contributions from both modulus 
(gravity) mediated contributions and the so-called anomaly mediated contributions, their relative size depending on a phenomenological parameter $\alpha$, defined in the next section. The anomaly-mediated SUSY breaking (AMSB) contributions[3] can be comparable because of the mass hierarchy

$$
m_{\text {moduli }} \gg m_{3 / 2} \gg m_{\mathrm{SUSY}}
$$

that develops. This hierarchy automatically alleviates phenomenological problems from late decaying moduli and gravitinos that could disrupt, for instance, the predictions of light element abundances from Big Bang nucleosynthesis. Aspects of the phenomenology of these models have recently been explored by several groups $4,4,5$, 6. In the notation of Ref. [5, which we adopt in this paper, in the limit $|\alpha| \rightarrow 0$ one obtains SSB terms which are pure AMSB with attendant tachyonic slepton squared masses. ${ }^{1}$ In the limit of large $|\alpha|$, one obtains dominantly modulus mediation (MM), possibly with nearly universal soft terms. For intermediate values of $|\alpha|$ of most interest to us, the problem of tachyonic sleptons is absent, and the phenomenology is the most novel. ${ }^{2}$ Following Ref. [4], we will refer to this scenario as the mixed modulus-anomaly mediated SUSY breaking (MM-AMSB) model.

Regardless of its theoretical motivation and stringy underpinnings, an examination of the phenomenology of the MM-AMSB framework is interesting in its own right. It represents a particular fusion of two well-studied models and can be regarded as a different, theoretically consistent and phenomenologically viable framework for the exploration of supersymmetry phenomenology. As we will see in the following, the SSB parameters depend on the so-called matter and gauge field modular weights that characterize the location of these fields in the extra spatial dimensions. In the absence of any specific string model compactification to select out a particular vacuum, we treat these as phenomenological numbers, different choices of which lead to quite different characteristics of the sparticle spectrum, and hence different SUSY phenomenology.

Several groups have begun the exploration of the phenomenology of MM-AMSB scenario [4, 5, 6]. Of particular importance in this regard is the nature of the lightest supersymmetric particle (LSP), which we will take to be the neutralino, since by (1.1), the gravitino is heavier than MSSM sparticles. First, this affects the topology of SUSY events at colliders: for instance, a higgsino-like neutralino LSP will couple more strongly to the third generation, thereby increasing the $b$-jet[7] and $\tau$-lepton multiplicity in SUSY events [8]. Second, the mass and composition of the LSP sensitively affect its annihilation rate in the early universe, and hence also the expected thermal dark matter LSP relic density that has now been determined at better than the $10 \%$ level by a study of the cosmic microwave background to be [9],

$$
\Omega_{C D M} h^{2}=0.111_{-0.01}^{+0.006}
$$

where we quote the value obtained by the WMAP collaboration by combining their three

\footnotetext{
${ }^{1}$ We warn the reader that $\alpha$ defined in Ref. [4] differs from the definition in Ref. 胞 that we use here by $\alpha_{\text {Ref. 团] }}=\frac{16 \pi^{2}}{\ln \left(M_{P} / m_{\frac{3}{2}}\right)} \frac{1}{\alpha_{\text {our }}}$.

${ }^{2}$ Of course, the hallmark feature of AMSB models, that the induced SSB parameters are scale invariant, no longer obtains.
} 
year result combined with the Sloan Digital Sky Survey data. ${ }^{3}$ This measurement serves as a stringent constraint on any model with a stable weakly interacting massive particle such as the neutralino in $R$-parity conserving SUSY models 10]. Third, the mass and composition of the neutralino sensitively determine the prospects for its detection in direct and indirect searches for dark matter [11]. The character of the neutralino LSP varies widely depending on the parameter $\alpha$, and also on the location of the gauge fields in the extra dimensions. Finally, the authors of Ref. [12 argue that, even for heavy top squarks, for some choices of MM-AMSB model parameters the value of $m_{H_{u}}^{2}\left(M_{\mathrm{GUT}}\right)$ is largely cancelled by its renormalization between $Q=M_{\mathrm{GUT}}$ and $Q=M_{Z}$; as a result, these models may have less fine-tuning relative to other frameworks.

The purpose of this paper is to map out the SUSY reach within the MM-AMSB model framework in regions of model parameter space where indirect constraints from rare $B$ and $B_{s}$ decays, from $(g-2)_{\mu}$, from the DM relic density, and from direct sparticle and Higgs boson searches are respected. Toward this end, we first map out the parameter space regions consistent with the WMAP constraint (1.2): agreement with (1.2) occurs due to a variety of mechanisms, depending on where we are in parameter space. We then delineate the SUSY reach of the CERN LHC and a $\sqrt{s}=0.5-1 \mathrm{TeV}$ linear $e^{+} e^{-}$collider in these regions. We also comment on particular characteristics of the SUSY collider signatures for selected model scenarios, and remark on the prospects for direct and indirect detection of the neutralino LSP within this framework. The remainder of this paper is organized as follows. We briefly review the KKLT construction, and highlight the characteristics of the SSB parameters within the MM-AMSB framework in Sec. 2. In Sec. 3, we examine the mass spectrum of the model for the case of zero modular weights for matter supermultiplets. We find a rather large magnitude for the $\mu$ parameter, so that the LSP is dominantly bino-like. ${ }^{4}$ The next-to-lightest supersymmetric particle (NLSP) is found to be either a top squark or a tau slepton. In this case, WMAP allowed regions are obtained where top squark or tau slepton co-annihilation effects act to suitably reduce the neutralino relic density. The WMAP allowed regions give rather low rates for direct and indirect detection of neutralino dark matter. We also estimate the reach of the CERN LHC and also a $\sqrt{s}=0.5-1 \mathrm{TeV}$ International Linear Collider (ILC). In our calculations, almost all the WMAP allowed region of parameter space should be accessible to LHC searches. In Sec. ઔ, we examine a case with non-zero modular weights (NZMW) for which the top squark can be more massive, so stop co-annihilation can no longer occur. In this case, we show that $A$ and $H$-funnel annihilation may be possible. Finally, in Sec. 5, we summarize our results and present some conclusions.

\footnotetext{
${ }^{3}$ The central value we use is almost unchanged from their earlier result based on the analysis of just the first year WMAP data. Our conclusions are insensitive to the precise value that we use.

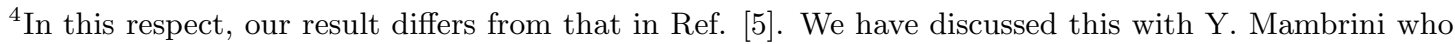
concurs with us that the discrepancy occurs because of an error in the sign convention for the $A$-parameter used in Ref. [5]. Once this is fixed, our results are in qualitative agreement.
} 


\section{MM-AMSB model and soft SUSY breaking parameters}

\subsection{The KKLT construction}

The KKLT construction [1] realizes metastable de Sitter vacua with all moduli stabilized. It breaks supersymmetry in a controlled way. In this construction, one first introduces nonzero fluxes in the Type IIB string theory compactified on a Calabi-Yau manifold. Due to the nonzero fluxes, the complex structure moduli and the dilaton are completely fixed. However, the size modulus $\hat{T}$ remains a flat direction and is still not fixed. To fix this, KKLT invoke non-perturbative effects, such as gaugino condensation on $D 7$ branes. At this stage, all moduli are fixed, but one ends up with supersymmetric vacua and negative vacuum energy. The final step in the construction is to include anti $D$-branes yielding the desired de-Sitter vacua (with positive vacuum energy) and breaking supersymmetry. Because of the presence of branes and fluxes, the models have generically warped compactifications. Due to the warping, the addition of the anti $D$-brane breaks supersymmetry by a very small amount.

Since the shape moduli are heavy enough to be integrated out, the low energy theory can be regarded as a broken supergravity theory of the size modulus $\hat{T}$. The Kähler potential for $\hat{T}$ is the no-scale type, and an exponential superpotential for it is induced by non-perturbative effects. Analysis [2] shows the modulus-mediated contribution to the SSB parameters is roughly

$$
\frac{F^{T}}{T+T^{*}}=\mathcal{O}\left(\frac{m_{3 / 2}}{\ln \left(M_{P} / m_{3 / 2}\right)}\right) \sim \frac{m_{3 / 2}}{4 \pi^{2}}
$$

where $T$ denotes the scalar component and $F^{T}$ the auxiliary component of the size modulus. To obtain the last equality, we assume $m_{3 / 2} \sim 1 \mathrm{TeV}$. Because of the additional suppression by the large logarithm, we see that the tree-level modulus-mediated contributions to MSSM SSB parameters can be comparable to the corresponding AMSB contribution, whose scale is given by,

$$
m_{\mathrm{soft}} \sim \frac{m_{3 / 2}}{8 \pi^{2}}
$$

The original KKLT proposal predicted the relative size of the modulus and AMSB contributions. It is, however, possible to generalize this picture so that the ratio of these contributions is determined by a phenomenological parameter $\alpha$ (that can have either

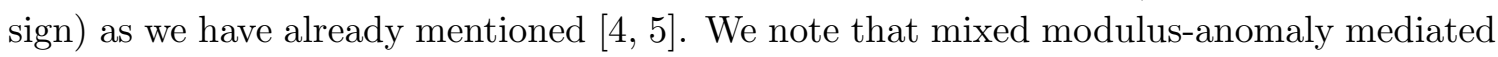
contributions to SSB parameters may also be seen in some of the "benchmark models" of Ref. [13], where references to the literature for their theoretical basis may be found.

The gauge kinetic functions and the Kähler potential for the visible matter superfields $\hat{Q}_{i}$ depend on their location in the extra dimensions. The gauge kinetic function is given by,

$$
f_{a}=\hat{T}^{\ell_{a}},
$$


where $a$ labels the gauge group and the power $\ell_{a}=1(0)$ for gauge fields on $D 7(D 3)$ branes. The Kähler potential for the matter fields is

$$
K=\sum_{i} \frac{1}{\left(\hat{T}+\hat{T}^{*}\right)^{n_{i}}} \hat{Q}_{i}^{*} \hat{Q}_{i}
$$

with the modular weights $n_{i}=0$ (1) for matter fields located on $D 7(D 3)$ branes; fractional values $n_{i}=1 / 2$ are also possible for matter living at brane intersections [4]. From the gauge kinetic functions, the Kähler potential and the superpotential, one can calculate visible field SSB parameters that are required for SUSY phenomenology.

\subsection{MM-AMSB model parameter space and soft terms}

The MM-AMSB model is completely specified by the parameter set,

$$
m_{3 / 2}, \alpha, \tan \beta, \operatorname{sign}(\mu), n_{i}, \ell_{a} .
$$

The mass scale for the SSB parameters is dictated by $m_{3 / 2}$, where $m_{3 / 2}$ is the gravitino mass. The parameter $\alpha$ gives the relative contributions of anomaly mediation and gravity mediation to the soft terms, $n_{i}$ are the modular weights of the visible sector matter fields, and $\ell_{a}$ appears in the gauge kinetic function. We see from (2.1) and (2.2) for $|\alpha|=\mathcal{O}(1)$ that the scale for the SSB parameters of the visible sector is $\sim \frac{m_{3 / 2}}{8 \pi^{2}}$.

More specifically, these SSB gaugino mass parameters, trilinear SSB parameters and sfermion mass parameters, all renormalized just below the unification scale (which we take to be $Q=M_{\mathrm{GUT}}$ ), are respectively given by,

$$
\begin{aligned}
M_{a} & =M_{s}\left(\ell_{a} \alpha+b_{a} g_{a}^{2}\right), \\
A_{i j k} & =M_{s}\left(-a_{i j k} \alpha+\gamma_{i}+\gamma_{j}+\gamma_{k}\right), \\
m_{i}^{2} & =M_{s}^{2}\left(c_{i} \alpha^{2}+4 \alpha \xi_{i}-\dot{\gamma}_{i}\right),
\end{aligned}
$$

where

$$
M_{s} \equiv \frac{m_{3 / 2}}{16 \pi^{2}},
$$

$b_{a}$ are the gauge $\beta$ function coefficients for gauge group $a$ and $g_{a}$ are the corresponding gauge couplings. The coefficients that appear in (2.6)-(2.8) are given by,

$$
\begin{gathered}
c_{i}=1-n_{i}, \\
a_{i j k}=3-n_{i}-n_{j}-n_{k},
\end{gathered}
$$

and

$$
\xi_{i}=\sum_{j, k} a_{i j k} \frac{y_{i j k}^{2}}{4}-\sum_{a} l_{a} g_{a}^{2} C_{2}^{a}\left(f_{i}\right) .
$$

Finally, $y_{i j k}$ are the superpotential Yukawa couplings, $C_{2}^{a}$ is the quadratic Casimir for the $\mathrm{a}^{\text {th }}$ gauge group corresponding to the representation to which the sfermion $\tilde{f}_{i}$ belongs, $\gamma_{i}$ is the anomalous dimension and $\dot{\gamma}_{i}=8 \pi^{2} \frac{\partial \gamma_{i}}{\partial \log \mu}$. Expressions for the last two quantities involving the anomalous dimensions are presented in the Appendix of Ref. [5], and will 
not be repeated here. For brevity, we will sometimes use the notation that $A_{t} \equiv A_{Q_{3} H_{u} u_{R}}$, $A_{b} \equiv A_{Q_{3} H_{d} d_{R}}$ and $A_{\tau} \equiv A_{L_{3} H_{d} e_{R}}$.

Throughout our study, we set $\ell_{a}=1$, but examine the phenomenology for various choices of modular weights, beginning with $n_{i}=0$ in the next section.

\section{MM-AMSB Model with zero modular weights}

\subsection{Soft SUSY breaking terms}

Following Ref. [5], we first examine the MM-AMSB model with modular weights $n_{i}=0$, and $\ell_{a}=1$. In this case, the contributions from modulus mediation are universal, and for large $\alpha$ the mass pattern reduces to that of minimal supergravity with $m_{0}=m_{1 / 2}=-A_{0} / 3$. The values of SSB parameters, renormalized at $Q=M_{\mathrm{GUT}}$, are plotted in Fig. 1 versus $\alpha$ for $m_{3 / 2}=11.5 \mathrm{TeV}, \tan \beta=10$ and $\mu>0$. We take $m_{t}=175 \mathrm{GeV}$ throughout this paper. In frame $a$ ), we show the gaugino masses and $A_{i}$-parameters, while in frame $b$ ) third generation sfermion and Higgs boson scalar mass parameters are shown as $\operatorname{sign}\left(m_{i}^{2}\right) \cdot \sqrt{\left|m_{i}^{2}\right|}$. We see from frame $a$ ) that for $\alpha=0$, the familiar pattern of AMSB gaugino masses results: $M_{1}>M_{2}$, while $M_{3}<0$. As $\alpha$ increases, all gaugino masses become positive. Moreover, because the differences $M_{1}-M_{2}$ and $M_{1}-M_{3}$ are independent of $\alpha$, the gaugino masses become essentially equal for large values of $|\alpha|$, as we had anticipated above. ${ }^{5}$ Since the GUT scale gaugino masses do not depend on the modular weights for matter fields, their behavior in frame a) also holds for models with non-zero modular weights considered in the next section.

For zero modular weights, the $A_{i}$ parameters would also be universal if the AMSB contributions were small relative to the modulus-mediated contributions, but instead they suffer some splitting. For $|\alpha| \gtrsim 2$, the universal modulus-mediated contributions dominate the AMSB contributions. As we shall see, the large $A_{t}$ term acts via $\mathrm{RG}$ running to suppress the scalar squared mass, $m_{\tilde{t}_{R}}^{2}$, so that the top squark $\tilde{t}_{1}$ is frequently the next-to-lightest SUSY particle (NLSP).

The SSB masses for third generation and Higgs scalars are shown in Fig. 1 $b$ ). For large values of $|\alpha|$, the $\alpha^{2}$ term in Eq. (2.8) dominates and we get the linear dependence seen in the figure. As the magnitude of $\alpha$ reduces, the other terms grow in importance leading to the curvature in the neighbourhood of $|\alpha| \lesssim 5$. We also see that for $\alpha$ close to zero, we reproduce the well-known tachyonic slepton squared masses of AMSB. This does not necessarily mean that we are in the wrong vacuum because we may expect large logarithmic corrections to the "tree level potential with parameters renormalized at the GUT scale". Indeed after renormalization group evolution to low scales, we see that over a portion of this range of $\alpha$ we find acceptable spectra at the weak scale. ${ }^{6}$ For moderate to

\footnotetext{
${ }^{5}$ The differences between gaugino masses would increase with $\alpha$ if the weights $\ell_{a}$ were dependent on $a$, but this would mean that the gauge fields of the different factors of the gauge group have different locations in the extra dimensions, precluding the possibility of Grand Unification.

${ }^{6}$ In the AMSB framework, $t$-squark masses are also negative at the GUT scale, but evolve to positive values at low scales. Negative soft masses for Higgs scalars have been considered previously in [14], while negative matter scalar soft masses have also been considered by Feng et al. 115.
} 
large values of $|\alpha|$, the moduli-mediated contributions dominate, and $m_{\tilde{t}_{L}}^{2}$ and $m_{\tilde{b}_{R}}^{2}\left(m_{\tilde{t}_{R}}^{2}\right)$ are the smallest of the squark soft breaking terms at the GUT scale for positive (negative) values of $\alpha$.

In Fig. 2 2 $a$ ), we illustrate the evolution of the three gaugino masses from $Q=M_{G U T}$ to $Q=M_{\text {weak }}$, for $\alpha=6, m_{3 / 2}=11.5 \mathrm{TeV}, \tan \beta=10$ and $\mu>0$. While the soft terms are ordered as $M_{1}>M_{2}>M_{3}$ at $M_{G U T}$, the RG evolution leads to the familiar weak scale ordering, $M_{3}>M_{2}>M_{1}$, expected in models with gaugino mass unification. As a result, unless $|\mu| \lesssim M_{1}$ (weak), the LSP is likely to be bino-like. Note, however, that the ratios of weak scale gaugino masses is now $M_{1}: M_{2}: M_{3} \sim 1: 1.3: 2.6$, and differs sharply from $M_{1}: M_{2}: M_{3} \sim 1: 2: 7$ in models with gaugino mass unification at the GUT scale, or in gauge-mediated SUSY breaking models. The presence of light gluinos in this framework will enhance the reach of hadron colliders over electron-positron colliders. The most striking feature of the figure is the well-understood phenomenon of "mirage unification" [6, 迆, where from a weak scale perspective, it appears that the gaugino masses unify at the intermediate scale $Q \sim 10^{11} \mathrm{GeV}$; gauge couplings, however, continue to unify at $Q=M_{\mathrm{GUT}}$.

In Fig. 2b), we show the evolution of the $A_{i}$ parameters versus $Q$ for the same parameter choice as in frame $a$ ). In this case, the evolution of $A_{t}$ is rather flat, while the magnitudes of $A_{b}$ and $A_{\tau}$ actually increase. The $A_{i}$ term RGEs read

$$
\begin{aligned}
\frac{d A_{t}}{d t} & =\frac{2}{16 \pi^{2}}\left(\sum_{i} c_{i} g_{i}^{2} M_{i}+6 f_{t}^{2} A_{t}+f_{b}^{2} A_{b}\right), \\
\frac{d A_{b}}{d t} & =\frac{2}{16 \pi^{2}}\left(\sum_{i} c_{i}^{\prime} g_{i}^{2} M_{i}+6 f_{b}^{2} A_{b}+f_{t}^{2} A_{t}+f_{\tau}^{2} A_{\tau}\right), \\
\frac{d A_{\tau}}{d t} & =\frac{2}{16 \pi^{2}}\left(\sum_{i} c_{i}^{\prime \prime} g_{i}^{2} M_{i}+3 f_{b}^{2} A_{b}+4 f_{\tau}^{2} A_{\tau}\right),
\end{aligned}
$$

where $c_{i}=\left(\frac{13}{15}, 3, \frac{16}{3}\right), c_{i}^{\prime}=\left(\frac{7}{15}, 3, \frac{16}{3}\right)$ and $c_{i}^{\prime \prime}=\left(\frac{9}{5}, 3,0\right)$. On the right hand side, the terms involving gauge couplings push the (already negative) $A_{i}$ parameters to more negative values, while the Yukawa terms push towards less negative values. The large top quark Yukawa coupling $f_{t}$ counterbalances the gauge terms to yield a nearly flat running for $A_{t}$, while the smaller $f_{b}$ and $f_{\tau}$ Yukawa couplings are not sufficient to counterbalance the push of the gauge terms. For negative values of $\alpha$, the gaugino mass parameters are negative, but $A$-parameters start off positive, and the cancellation between the gauge and Yukawa contributions to the evolution of $A$-parameters persists.

The weak scale values of the gaugino masses are shown in Fig. 3 for the same slice of parameter space as in Fig. 2. The gaugino mass parameters are essentially independent of matter modular weights (which enter only via sfermion masses either via two-loop terms in the RGEs, or via sparticle decoupling). The striking feature of this figure is that for $\alpha \sim 2.5$, the mirage unification now occurs essentially at the weak scale. Close to this value of $\alpha, M_{1}$ (weak) $=M_{2}$ (weak) so that it is possible that mixed wino dark matter (MWDM), if 
allowed within this framework by other constraints, is a viable DM candidate 16]. Likewise, for small and negative values of $\alpha, M_{1}$ (weak) $\sim-M_{2}$ (weak), and bino-wino coannihilation (BWCA) offers a viable possibility for DM in agreement with Eq. (1.2) [17]. We should mention that, depending on modular weights, different ranges of $\alpha$ are excluded because of theoretical constraints: either electroweak symmetry is not properly broken, or the LSP is a charged particle. These ranges are shown for the model with zero modular weights on the upper scale, and for a model with the choice $n_{H_{u}}=n_{H_{d}}=1, n_{\text {matter }}=\frac{1}{2}$ considered in the next section.

Returning to the RGE of SSB parameters, in Fig. 4 4 a) we show the evolution of first generation scalar soft breaking masses. Here, we see that the GUT scale mass ordering $m_{e_{R}}^{2}>m_{e_{L}}^{2}>m_{d_{R}}^{2}>m_{u_{R}}^{2}>m_{u_{L}}^{2}$ (but with rather small splittings) becomes essentially inverted at the weak scale, mainly because of the large evolution of squark masses on account of SUSY QCD interactions. The mirage unification of scalar soft terms at $Q \sim 10^{11}$ $\mathrm{GeV}$ is also evident. In Fig. $4 b$ ) we show the evolution of third generation and Higgs boson soft scalar mass parameters. Since their evolution depends on Yukawa couplings, the mirage unification no longer obtains: notice, however, that the $H_{d}$ and $\tilde{b}_{R}$ mass parameters (for which the Yukawa couplings are small) do intersect close to the mirage unification scale in frame $a$ ). We also see that $m_{\tilde{t}_{R}}^{2}$ evolves to much lower values than other squark masses and, in this case, is not very different from the corresponding slepton and wino mass parameters. This effect, along with large mixing in the top squark mass matrix, leads to $m_{\tilde{t}_{1}}$ being the NLSP in the MM-AMSB model with zero modular weights and low $\tan \beta$.

\subsection{Mass spectrum}

Once the soft SUSY breaking terms are stipulated at $Q=M_{G U T}$, then we use Isajet v7.74 18 to compute the corresponding weak scale mass spectrum. In Fig. 5, we show sparticle masses and the weak scale $\mu$ parameter vs. $\alpha$ for $m_{3 / 2}=11.5 \mathrm{TeV}, \mu>0$ and $a$ ) $\tan \beta=10$ and $b) \tan \beta=30$. The red-shaded region is excluded by lack of REWSB, and the blue-shaded region gives a charged or colored $\left(\tilde{t}_{1}\right.$ or $\left.\tilde{\tau}_{1}\right)$ LSP, so that viable mass spectra are only achieved for $\alpha \gtrsim 5.5$ or $\alpha \lesssim-1.2$ The lines end at large values of $|\alpha|$ because electroweak symmetry is not correctly broken. Since $\left|M_{1}\right|<M_{2}<M_{3}$ and $|\mu| \gg M_{1}$, the LSP $\widetilde{Z}_{1}$ is bino-like, and the corresponding relic density is typically large and beyond the WMAP bound. However, we see in frame a) that at low $\alpha \sim 6, m_{\tilde{t}_{1}} \sim m_{\widetilde{Z}_{1}}$ so that top squark co-annihilation is possible, which can act to reduce the relic density, while for $\alpha$ just smaller than -2 , the BWCA mechanism could be operative as anticipated above. For this choice of modular weights and $\tan \beta$, MWDM is not possible, because $\tilde{t}_{1}$ becomes the LSP for the required value of $\alpha$. In the case of frame $b$ ) with a larger value of $\tan \beta=30$, we find for low positive $\alpha$ that $m_{\tilde{\tau}_{1}} \sim m_{\tilde{t}_{1}} \sim m_{\widetilde{Z}_{1}}$, so that top and stau co-annihilation is possible, while for negative values of $\alpha$, it appears that co-annihilation is precluded for at least this choice of parameters.

In Fig. 6, we show sparticle masses and the $\mu$ parameter vs. $m_{3 / 2}$ for fixed $\alpha=6$ and $\mu>0$ and the same two choices of $\tan \beta$. Along this strip, the neutralino is again bino-like resulting in too large a relic density. The exception is at low $m_{3 / 2} \sim 8 \mathrm{TeV}$ where in frame a) for $\tan \beta=10$ we again find $m_{\tilde{t}_{1}} \sim m_{\widetilde{Z}_{1}}$, so that top squark co-annihilation is possible. 
In frame $b$ ) with $\tan \beta=30$, we find that for low values of $m_{3 / 2}, m_{\tilde{\tau}_{1}} \sim m_{\widetilde{Z}_{1}}$ so that tau slepton co-annihilation acts to reduce the relic density (to too low a value), along with contributions from the Higgs-funnel annihilation.

In Fig. 0, we plot sparticle masses as well as $\mu$ versus $\tan \beta$ for $\alpha=6, m_{3 / 2}=11.5$ $\mathrm{TeV}$ and $a) \mu>0$ and $b) \mu<0$. We see in this case that the allowed parameter space only extends up to $\tan \beta \sim 31$ for $\mu>0$ and $\tan \beta \sim 38$ for $\mu<0$. In both cases, the $\tilde{\tau}_{1}$ becomes the LSP at the upper limit on $\tan \beta$, so in this region, $\tilde{\tau}_{1}-\widetilde{Z}_{1}$ co-annihilation is again possible. Note that the value of $m_{A}$ is a decreasing function of $\tan \beta$, and for $\mu<0$ (which, for positive values of $\alpha$, is disfavored by the $(g-2)_{\mu}$ anomaly), a region around $\tan \beta \sim 25$ also becomes WMAP allowed, since here $A$-funnel annihilation can occur, since $2 m_{\widetilde{Z}_{1}} \sim m_{A}$. The situation is illustrated in Fig. 8, where we plot $\Omega_{\widetilde{Z}_{1}} h^{2} v s$. $\tan \beta$ for the same parameters as in Fig. 7 .

In Fig. 9, we show the entire $\alpha$ vs. $m_{3 / 2}$ parameter space plane for $\mu>0$ and $a$ ) $\tan \beta=10$ and $b) \tan \beta=30$. In the white region, either radiative electroweak symmetry breaking fails or there are sparticles with masses below the lower limits from LEP 2 . Points with a turquiose square are excluded because $\tilde{t}_{1}$ is the LSP, while those with a magenta square are excluded because the LSP is $\tilde{\tau}_{1}$. Points denoted by blue dots are theoretically allowed spectra, but give $\Omega_{\widetilde{Z}_{1}} h^{2}>0.5$, and so are excluded by the relic density measurement. Points denoted by a green $\times$ are also excluded, since $0.13<\Omega_{\widetilde{Z}_{1}} h^{2}<0.5$. Finally, points denoted by a red + give $\Omega_{\widetilde{Z}_{1}} h^{2}<0.13$ and are allowed by the relic density constraint. (Remember that either the CDM could consist of several components, or there may be a non-thermal component of DM.) The allowed region extends from very high values of $m_{3 / 2}>60 \mathrm{TeV}$ with $\alpha \sim 5.6$ to very low values of $m_{3 / 2}<3 \mathrm{TeV}$ for $\alpha>10$; for $\alpha<0$, the allowed region extends between $20 \mathrm{TeV} \lesssim m_{3 / 2} \lesssim 35 \mathrm{TeV}$. For positive values of $\alpha$, we have the bulk of the red + s right next to the " $\tilde{t}$ LSP" region, so that the correct relic density is attained via LSP co-annihilation with $\tilde{t}_{1}$. The few red $+\mathrm{s}$ to the left of the turquoise $\tilde{t}_{1}$ LSP region at low positive $\alpha$ and large $m_{3 / 2}$ are where we have a higgsino-like LSP because $M_{1} \sim M_{2} \sim M_{3}$, and the low value of $M_{3}$ leads to a small value of $\mu$ [19, 20, 21]. We do not see a region of MWDM where $M_{1}$ (weak) $\sim M_{2}$ (weak) since the values of $\alpha$ where this would have occurred lead to $\tilde{t}_{1}$ as the LSP. Turning to $\alpha<0$ in frame $a$ ), we see a region consistent with relic density constraints where $\alpha \sim-1.5$ and $m_{3 / 2} \sim 20-35 \mathrm{TeV}$. In this region, we have $M_{1}$ (weak) $\sim-M_{2}$ (weak) as we anticipated earlier. For the most negative values of $\alpha \sim-1.63$, the relic density is in accord with (1.2) via BWCA [17], while for somewhat less negative values of $\alpha$, the LSP becomes wino-like, and an additional source of DM is needed to saturate the measured value of DM relic density. For the larger value of $\tan \beta$ in frame $b$ ), the radiative EWSB mechanism fails for the low negative values of $\alpha$, so that the corresponding region is absent. ${ }^{7}$

Several sparticle masses together with values of various observables for four sample points are listed in Table 1. Point 1 with large $m_{3 / 2}=40 \mathrm{TeV}$ and $\alpha=5.5$ is characterized by a rather heavy sparticle mass spectrum, but where $m_{\tilde{t}_{1}}=1076 \mathrm{GeV}$ while $m_{\widetilde{Z}_{1}}=979$

\footnotetext{
${ }^{7}$ With large negative values of $\alpha$, the gaugino masses would be negative and solutions with $\mu>0$ would be disfavoured because the SUSY contributions to the muon anomalous magnetic moment would be negative. It may be of interest to examine this part of the parameter space for solutions with $\mu<0$.
} 
$\mathrm{GeV}$. The $\tilde{t}_{1}-\widetilde{Z}_{1}$ mass gap is sufficiently low that top squark co-annihilation can efficiently reduce the neutralino relic density $\Omega_{\widetilde{Z}_{1}} h^{2}$ to WMAP allowed levels. The rather heavy sparticle mass spectrum gives rise to only tiny contributions to $B F(b \rightarrow s \gamma)$ and $\Delta a_{\mu}$ so that these quantities would be expected to be measured at nearly their SM values. Point 2 is taken at larger $\alpha=8$ but lower $m_{3 / 2}=4.6 \mathrm{TeV}$. This case gives rise to a rather light sparticle mass spectrum, although the $\tilde{t}_{1}$ is again the NLSP. A combination of bulk annihilation through $t$-channel sfermion exchange and top squark co-annihilation act to reduce the relic density to WMAP allowed levels. The light top squark leads to large non-standard contributions to $B F(b \rightarrow s \gamma)$, and in this case a very low branching fraction, below its experimental lower bound would be expected. Point 3 is taken with $\tan \beta=30$, $m_{3 / 2}=19 \mathrm{TeV}$ and $\alpha=6$. While sparticle masses tend to range between 500-1000 GeV, we find a stau NLSP with $m_{\tilde{\tau}_{1}}=515 \mathrm{GeV}$ and $m_{\widetilde{Z}_{1}}=480.2 \mathrm{GeV}$. In this case, it is mainly stau co-annihilation that acts to reduce the relic density to WMAP allowed levels. This point gives a $B F(b \rightarrow s \gamma)=2.3 \times 10^{-4}$, somewhat at the lower end of the range but probably acceptable if we include theoretical uncertainties for this value of $\tan \beta$. Finally, we consider point 4 in the region with negative $\alpha$ where we get the required relic density via the BWCA mechanism: here, we take $\alpha=-1.635, m_{3 / 2}=25 \mathrm{TeV}$ and $\tan \beta=10$. We see that squark, gluino and Higgs boson masses are qualitatively similar to those for Point 3, but the uncoloured sparticles are significantly lighter. The LSP is a bino but the chargino and $\widetilde{Z}_{2}$ are dominantly wino-like and close in mass to the LSP. Since the winos can annihilate efficiently, as long as thermal equilibrium is maintained, the LSP density is correspondingly reduced. The relatively light $t$-squark and chargino give a significant contribution to $B F(b \rightarrow s \gamma)$, which is somewhat on the high side. The SUSY contribution to the muon magnetic moment, though negative, is modest because $\tan \beta$ is only 10 .

A rather general feature of the case of the zero modular weight MM-AMSB model with positive $\alpha$ is the existence of a top squark with a mass comparable to $m_{\widetilde{Z}_{1}}$ and a bino-dominated LSP. The very light top squark is a consequence of the large $A_{t}$ parameter coupled with a value of $M_{3}$ which is reduced relative to expectations from models with gaugino mass unification (e.g. mSUGRA). Thus, over much of the $\alpha v s . m_{3 / 2}$ parameter plane, $\tilde{t}_{1}-\widetilde{Z}_{1}$ co-annihilation leads to a relic density compatible with the WMAP determination. As remarked in Sec. 1, this is in contrast with the result in Ref. [5], where a low value of $|\mu|$, and consequently, mixed higgsino dark matter (MHDM) is obtained because the weak scale $\left|M_{3}\right|$ is reduced relative to $\left|M_{1}\right|$ and $\left|M_{2}\right| \mid 19$, 20, 21]. While we do find a reduction in $|\mu|$, it is not large enough to change the $\widetilde{Z}_{1}$ from being nearly pure bino to being MHDM. ${ }^{8}$ Instead, we find that much of the phenomenology derives from the rather large magnitude of the $A_{t}$ parameter in this framework. This, in turn, occurs because apart from splitting due to the AMSB terms in (2.7), the GUT scale magnitude of $A_{t}$ is roughly three times the value of GUT scale gaugino and scalar masses, and its evolution is approximately flat, resulting in a large value also at the weak scale. Now recall that the

\footnotetext{
${ }^{8}$ The distinction between a bino LSP and MHDM is especially important for direct and indirect searches for relic DM.
} 


\begin{tabular}{|c|c|c|c|c|}
\hline parameter & Point 1 & Point 2 & Point 3 & Point 4 \\
\hline$\alpha$ & 5.5 & 8 & 6 & -1.635 \\
\hline$m_{3 / 2}(\mathrm{TeV})$ & 40 & 4.6 & 19 & 25 \\
\hline $\tan \beta$ & 10 & 10 & 30 & 10 \\
\hline$\mu$ & 1753.7 & 371.7 & 967.5 & 961.0 \\
\hline$m_{\tilde{g}}$ & 2256.2 & 475.5 & 1257.3 & 1154.4 \\
\hline$m_{\tilde{u}_{L}}$ & 2273.5 & 470.2 & 1261.3 & 1129.4 \\
\hline$m_{\tilde{t}_{1}}$ & 1076.5 & 161.4 & 594.4 & 687.2 \\
\hline$m_{\tilde{b}_{1}}$ & 1871.4 & 395.7 & 998.5 & 952.5 \\
\hline$m_{\tilde{e}_{L}}$ & 1536.3 & 270.9 & 806.9 & 369.2 \\
\hline$m_{\tilde{e}_{R}}$ & 1438.8 & 247.4 & 749.9 & 275.7 \\
\hline$m_{\tilde{\tau}_{1}}$ & 1397.8 & 232.0 & 515.0 & 250.4 \\
\hline$m_{\widetilde{W}_{1}}$ & 1249.7 & 182.9 & 632.6 & 141.5 \\
\hline$m_{\widetilde{Z}_{2}}$ & 1245.0 & 183.3 & 630.8 & 141.2 \\
\hline$m_{\widetilde{Z}_{1}}$ & 979.1 & 132.3 & 480.2 & 118.8 \\
\hline$m_{A}$ & 2326.7 & 445.9 & 1076.2 & 987.4 \\
\hline$m_{h}$ & 124.4 & 114.1 & 122.6 & 116.9 \\
\hline$\Omega_{\widetilde{Z}_{1}} h^{2}$ & 0.12 & 0.10 & 0.11 & 0.11 \\
\hline$B F(b \rightarrow s \gamma)$ & $3.3 \times 10^{-4}$ & $9.8 \times 10^{-5}$ & $2.3 \times 10^{-4}$ & $4.0 \times 10^{-4}$ \\
\hline$\Delta a_{\mu}$ & $6.1 \times 10^{-11}$ & $20.1 \times 10^{-10}$ & $6.5 \times 10^{-10}$ & $-2.2 \times 10^{-10}$ \\
\hline$B F\left(B_{s} \rightarrow \mu^{+} \mu^{-}\right)$ & $3.9 \times 10^{-9}$ & $4.1 \times 10^{-9}$ & $5.1 \times 10^{-9}$ & $3.8 \times 10^{-9}$ \\
\hline$\sigma_{s c}\left(\widetilde{Z}_{1} p\right)$ & $7.9 \times 10^{-11} \mathrm{pb}$ & $5.0 \times 10^{-9} \mathrm{pb}$ & $2.6 \times 10^{-10} \mathrm{pb}$ & $4.6 \times 10^{-11} \mathrm{pb}$ \\
\hline$\Phi^{\mu}\left(k m^{-2} y r^{-1}\right)$ & $4 \times 10^{-5}$ & 2.61 & 0.03 & $10^{-4}$ \\
\hline$\Phi^{\gamma}\left(c m^{-2} s^{-1}\right)$ & $\begin{array}{c}3.2 \times 10^{-11} \\
\left(1.6 \times 10^{-15}\right)\end{array}$ & $\begin{array}{c}2.0 \times 10^{-8} \\
\left(1.0 \times 10^{-13}\right)\end{array}$ & $\begin{array}{c}4.9 \times 10^{-9} \\
\left(2.5 \times 10^{-13}\right)\end{array}$ & $\begin{array}{l}2.2 \times 10^{-10} \\
\left(1.0 \times 10^{-14}\right)\end{array}$ \\
\hline$\Phi^{e^{+}}\left(\mathrm{GeV}^{-1} \mathrm{~cm}^{-2} s^{-1} s r^{-1}\right)$ & $\begin{array}{l}1.0 \times 10^{-12} \\
\left(2.1 \times 10^{-13}\right)\end{array}$ & $\begin{array}{l}1.3 \times 10^{-10^{\prime}} \\
\left(3.1 \times 10^{-11}\right)\end{array}$ & $\begin{array}{l}1.0 \times 10^{-10} \\
\left(2.3 \times 10^{-11}\right)\end{array}$ & $\begin{array}{c}2.1 \times 10^{-11} \\
\left(4.7 \times 10^{-12}\right)\end{array}$ \\
\hline$\Phi^{\bar{p}}\left(G e V^{-1} c^{-2} s^{-1} s r^{-1}\right)$ & $\begin{array}{l}4.9 \times 10^{-12} \\
\left(2.8 \times 10^{-13}\right)\end{array}$ & $\begin{array}{l}1.7 \times 10^{-9} \\
\left(9.6 \times 10^{-11}\right)\end{array}$ & $\begin{array}{l}7.0 \times 10^{-10^{\prime}} \\
\left(3.9 \times 10^{-11}\right)\end{array}$ & $2.9 \times 10^{-12}$ \\
\hline$\Phi^{\bar{D}}\left(G e V^{-1} \mathrm{~cm}^{-2} s^{-1} s r^{-1}\right)$ & $\begin{array}{l}1.6 \times 10^{-15} \\
\left(1.4 \times 10^{-16}\right)\end{array}$ & $\begin{array}{l}2.0 \times 10^{-12} \\
\left(1.7 \times 10^{-13}\right)\end{array}$ & $\begin{array}{l}2.9 \times 10^{-13} \\
\left(2.4 \times 10^{-14}\right)\end{array}$ & $\begin{array}{l}5.2 \times 10^{-15} \\
\left(4.4 \times 10^{-16}\right)\end{array}$ \\
\hline
\end{tabular}

Table 1: Masses and parameters in GeV units for four case studies of the MM-AMSB model with zero modular weights. Also shown are predictions for low energy observables, together with cross sections and fluxes germane to direct and indirect searches for dark matter. In all cases, we take $m_{t}=175 \mathrm{GeV}$ and $\mu>0$. The halo annihilation rates use the Adiabatically Contracted N03 Halo model, while the values in parenthesis use the Burkert halo profile.

RGEs for Higgs and squark SSB mass parameters have the form,

$$
\begin{aligned}
& \frac{d m_{H_{d}}^{2}}{d t}=\frac{2}{16 \pi^{2}}\left(-\frac{3}{5} g_{1}^{2} M_{1}^{2}-3 g_{2}^{2} M_{2}^{2}-\frac{3}{10} g_{1}^{2} S+3 f_{b}^{2} X_{b}+f_{\tau}^{2} X_{\tau}\right), \\
& \frac{d m_{H_{u}}^{2}}{d t}=\frac{2}{16 \pi^{2}}\left(-\frac{3}{5} g_{1}^{2} M_{1}^{2}-3 g_{2}^{2} M_{2}^{2}+\frac{3}{10} g_{1}^{2} S+3 f_{t}^{2} X_{t}\right), \\
& \frac{d m_{Q_{3}}^{2}}{d t}=\frac{2}{16 \pi^{2}}\left(-\frac{1}{15} g_{1}^{2} M_{1}^{2}-3 g_{2}^{2} M_{2}^{2}-\frac{16}{3} g_{3}^{2} M_{3}^{2}+\frac{1}{10} g_{1}^{2} S+f_{t}^{2} X_{t}+f_{b}^{2} X_{b}\right),
\end{aligned}
$$




$$
\begin{aligned}
& \frac{d m_{\tilde{t}_{R}}^{2}}{d t}=\frac{2}{16 \pi^{2}}\left(-\frac{16}{15} g_{1}^{2} M_{1}^{2}-\frac{16}{3} g_{3}^{2} M_{3}^{2}-\frac{2}{5} g_{1}^{2} S+2 f_{t}^{2} X_{t}\right), \\
& \frac{d m_{\tilde{b}_{R}}^{2}}{d t}=\frac{2}{16 \pi^{2}}\left(-\frac{4}{15} g_{1}^{2} M_{1}^{2}-\frac{16}{3} g_{3}^{2} M_{3}^{2}+\frac{1}{5} g_{1}^{2} S+2 f_{b}^{2} X_{b}\right),
\end{aligned}
$$

where

$$
\begin{aligned}
X_{t} & =m_{Q_{3}}^{2}+m_{\tilde{t}_{R}}^{2}+m_{H_{u}}^{2}+A_{t}^{2} \\
X_{b} & =m_{Q_{3}}^{2}+m_{\tilde{b}_{R}}^{2}+m_{H_{d}}^{2}+A_{b}^{2} \\
X_{\tau} & =m_{L_{3}}^{2}+m_{\tilde{\tau}_{R}}^{2}+m_{H_{d}}^{2}+A_{\tau}^{2}, \text { and } \\
S & =m_{H_{u}}^{2}-m_{H_{d}}^{2}+\operatorname{Tr}\left[\mathbf{m}_{Q}^{2}-\mathbf{m}_{L}^{2}-2 \mathbf{m}_{U}^{2}+\mathbf{m}_{D}^{2}+\mathbf{m}_{E}^{2}\right] .
\end{aligned}
$$

The large $A_{t}, A_{b}$ and $A_{\tau}$ parameters mean that the corresponding $X_{t}, X_{b}$ and $X_{\tau}$ parameters are also large. The $f_{t}^{2} X_{t}$ term in $d m_{H_{u}}^{2} / d t$ acts to drive $m_{H_{u}}^{2}$ to large negative values (this is the well-known REWSB mechanism). A large negative value of $m_{H_{u}}^{2}$ leads to a large $\mu$ value via the scalar potential minimization condition:

$$
\mu^{2}=\frac{m_{H_{d}}^{2}-m_{H_{u}}^{2} \tan ^{2} \beta}{\left(\tan ^{2} \beta-1\right)}-\frac{M_{Z}^{2}}{2} \sim-m_{H_{u}}^{2},
$$

where the last equality follows as long as $\tan \beta$ is not very close to 1 , and $\left|m_{H_{u}}^{2}\right| \gg M_{Z}^{2}$. Thus, even though a lower $M_{3}$ value acts to reduce $|\mu|$, the large $A_{t}$ parameter acts to increase it. Moreover, the large $X_{t}$ parameter also acts to suppress $m_{\tilde{t}_{R}}^{2}$ (and $m_{Q_{3}}^{2}$ ) via RG running. Finally, the large value of $A_{t}$ results in a large intragenerational top squark mixing, and further reduces the value of $m_{\tilde{t}_{1}}$.

We thus understand why the MM-AMSB model spectrum, for zero modular weights and positive $\alpha$, is characterized by a bino-like LSP $\widetilde{Z}_{1}$, but with either a $\tilde{t}_{1}$ NLSP at lower values of $\tan \beta$, or a $\tilde{\tau}_{1}$ NLSP at high $\tan \beta$. When the mass gap $m_{\tilde{t}_{1}}-m_{\widetilde{Z}_{1}}$ or $m_{\tilde{\tau}_{1}}-m_{\widetilde{Z}_{1}}$ is low enough, then co-annihilation can act reliably to reduce the neutralino relic density to WMAP allowed levels. Furthermore, in the neighborhood of Point 2, for instance, one can readily find cases of light $\tilde{t}_{1}$ in this model with $m_{\tilde{t}_{1}}<m_{t}$ with $m_{h}<120 \mathrm{GeV}$. These are among some of the important conditions required for successful electroweak baryogenesis in the MSSM[22].

\subsection{Prospects for colliders searches and DM search experiments}

If SUSY is realized as in the MM-AMSB model, it is expected that sparticle pair production will occur at observable rates at the CERN LHC. Indeed, since for positive but not too large values of $\alpha, M_{3}$ (weak) is smaller than its value in models with universal gaugino masses, gluinos (and, via renormalization, also squarks) will be rather more accessible at hadron colliders within this framework. The reach of the LHC for SUSY in the mSUGRA model has been computed in Ref. [23]. In these studies, the SUSY production cross sections are typically dominated by gluino and squark pair production, followed by cascade decays to a variety of multijet $+E_{T}^{\text {miss }}$ plus (multi)-isolated lepton final states. The SUSY reach of the LHC depends mainly on the gluino and squark masses, and is relatively insensitive 
to the particular cascade decay modes which are active (the details of the cascade decay modes are much more important for sparticle mass reconstruction and for arriving at the underlying model parameters). When $m_{\tilde{q}} \simeq m_{\tilde{g}}$, then the LHC reach with $100 \mathrm{fb}^{-1}$ of integrated luminosity was found to be $m_{\tilde{g}} \sim 3 \mathrm{TeV}$, while in the case where $m_{\tilde{q}} \gg m_{\tilde{g}}$, the LHC reach extends to $m_{\tilde{g}} \sim 1.8 \mathrm{TeV}$.

We expect the LHC reach in the MM-AMSB model to depend mainly on $m_{\tilde{q}}$ and $m_{\tilde{g}}$ as well, and not on the particular cascade patterns. Thus, to obtain the reach within this framework, we simply digitize the mSUGRA reach contours evaluated in the last of the papers in Ref. [23] in terms of $m_{\tilde{g}}$ and $m_{\tilde{q}}$, and map them onto the $\alpha$ vs. $m_{3 / 2}$ plane of the ZMW model in Fig. 9. It turns out that for positive $\alpha, m_{\tilde{g}} \simeq m_{\tilde{q}}\left(\right.$ while $\left.m_{\tilde{t}_{1}} \sim \frac{1}{3}-\frac{1}{2} m_{\tilde{q}}\right)$ throughout the parameter half plane, so the LHC reach extends essentially along the $m_{\tilde{g}} \sim 3$ $\mathrm{TeV}$ contour line. The $100 \mathrm{fb}^{-1} \mathrm{LHC}$ reach thus obtained is shown as the solid red contour in Fig. 9. The contour covers all WMAP allowed region when $\alpha$ assumes large values, but only reaches up to $m_{3 / 2} \sim 53 \mathrm{TeV}$ for $\alpha \sim 5.8$. If $\alpha<0$, the gluino and squark masses in the allowed region are well within the reach of the LHC, so that the contour extends essentially to the boundary of this allowed region.

We also evaluate the reach of a $\sqrt{s}=0.5 \mathrm{TeV}(1 \mathrm{TeV})$ linear $e^{+} e^{-}$collider (LC), where the contour is determined via the kinematic limits of $0.25(0.5 \mathrm{TeV})$ for either chargino, stau or stop pair production. The corresponding reaches are shown by the blue $(0.5 \mathrm{TeV})$ and green $(1 \mathrm{TeV})$ contours.

In the case studies illustrated in Table 1, we note that Point 1 is an example of an MM-AMSB model which should yield observable signals at the LHC via cascade decay signatures. Gluino and squark pair production, with $m_{\tilde{g}} \simeq m_{\tilde{q}} \simeq 2.3 \mathrm{TeV}$, will occur at the LHC with a cross section of several $\mathrm{fb}$. The gluinos mainly decay via two body decays to third generation quarks and squarks, whereas $\tilde{q}_{L}\left(\tilde{q}_{R}\right)$ mainly decay via $\tilde{q}_{L} \rightarrow q^{\prime} \widetilde{W}_{1}, q \widetilde{Z}_{2}$ $\left(\tilde{q}_{R} \rightarrow \widetilde{Z}_{1}\right)$, while $\widetilde{W}_{1} \rightarrow \tilde{t}_{1} b$ and $\widetilde{Z}_{2} \rightarrow h \widetilde{Z}_{1}$. Because the mass difference $m_{\tilde{t}_{1}}-m_{\widetilde{Z}_{1}}$ is small, the daughter $\tilde{t}_{1}$ will decay via $\tilde{t}_{1} \rightarrow c \widetilde{Z}_{1}$ : we expect the competing $\tilde{t}_{1} \rightarrow b W \widetilde{Z}_{1}$ decay will be more strongly suppressed by phase space. Gluino and squark production thus leads to events with $2-4$ very hard jets plus $E_{T}^{\text {miss }}$, with an enrichment of $b$-jets, with a not especially large multiplicity of isolated leptons.

For Point 2, the very light sparticle mass spectrum will provide enormous signal rates of order $10^{5} \mathrm{fb}$, with many multilepton states and, perhaps, several dilepton mass edges evident. In this case, $\widetilde{Z}_{2} \rightarrow e^{+} e^{-} \widetilde{Z}_{1}$ at an enhanced branching fraction level of $5.3 \%$. Once again, gluinos mostly decay to third generation quarks and squarks, but this time, the unusual feature is that the light chargino which is abundantly produced via cascade decays of $\tilde{q}_{L}$ mostly decays via $\widetilde{W}_{1} \rightarrow \tilde{t}_{1} b$ ! Indeed, since $m_{\tilde{t}_{1}}$ is only $161 \mathrm{GeV}$, it may also be possible to search for $\tilde{t}_{1}$ at the Fermilab Tevatron [24].

Point 3 should also lead to readily observable signals at the LHC. Once again gluinos decay to third generation particles and sparticles, whereas first generation squark decays are as for Point 1. Since $\tilde{\tau}_{1}$ is relatively light, production of charginos and neutralinos (either directly, or via cascade decays of $\tilde{q}_{L}$ ) lead to events rich in tau jets from $\widetilde{Z}_{2} \rightarrow \tau \tilde{\tau}$ and $\widetilde{W}_{1} \rightarrow \tilde{\tau}_{1} \nu_{\tau}$ decays, though it should be kept in mind that $B F\left(\widetilde{W}_{1} \rightarrow \tilde{t}_{1} b\right)=47 \%$. 
The light $\tilde{t}_{1}$-squark for $\alpha>0$ results in events rich in $b$-jets, as we saw for Points $1-3$ above. Turning to Point 4 with $\alpha<0$, the decay patterns of gluinos and squarks are similar to those for Points $1-3$. The difference is that now, because $\tilde{t}_{1}$ is significantly heavier than $\widetilde{Z}_{1}, \tilde{t}_{1} \rightarrow t \widetilde{Z}_{1}, b \widetilde{W}_{1}$ and even $\tilde{t}_{1} \rightarrow t \widetilde{Z}_{2}$. The unusual features of this scenario are that $\widetilde{Z}_{2}$ dominantly decays to $\widetilde{W}_{1}+f \bar{f}^{\prime}$ where $f, f^{\prime}$ are either the quarks of the first two generations or any of the leptons and neutrinos, and that the chargino mainly decays leptonically via three body decays with decays $\widetilde{W}_{1} \rightarrow \tau \nu_{\tau} \widetilde{Z}_{1}$ having a branching fraction of $56 \%$, while the corresponding decays to each of the first two generations each occurring $16 \%$ of the time.

Points 1 and 3, while observable at LHC, have no visible two body sparticle or nonStandard Model Higgs boson processes that will be accessible at even a $1 \mathrm{TeV}$ LC. On the other hand, for Point 2 there should be $\widetilde{W}_{1}^{+} \widetilde{W}_{1}^{-}, \widetilde{Z}_{1} \widetilde{Z}_{2}$ and $\tilde{t}_{1} \overline{\tilde{t}}_{1}$ signals at a $\sqrt{s}=0.5$ $\mathrm{TeV}$ LC, and possibly even a $\tilde{\tau}^{+} \tilde{\tau}^{-}$signal. Other sleptons, the additional Higgs bosons and also squarks will be accessible at $\sqrt{s}=1 \mathrm{TeV}$. For Point 4 , only $\widetilde{W}_{1}$ and $\widetilde{Z}_{2}$ will be kinematically accessible at a $\sqrt{s}=0.5 \mathrm{TeV}$ machine; however, since the mass gaps between these and $\widetilde{Z}_{1}$ is small, specialized analyses [25] will be necessary to extract the signal. All the sleptons and sneutrinos should be readily accessible at an $e^{+} e^{-}$collider operating at $\sqrt{s}=1 \mathrm{TeV}$.

Turning to direct and indirect detection of dark matter signals, we expect these to be in general rather low in this framework, primarily because the LSP is bino-like, and co-annihilation is usually needed to reduce the relic density. We have computed direct (using Isatools 26]) and indirect detection rates (using DarkSUSY 27]) for the four case studies in Table 1, and list these in the last few rows. Second generation direct detection experiments such as CDMS2 expect to probe spin-independent neutralino-proton scattering cross sections down to $\sim 3 \times 10^{-8} \mathrm{pb}$, while Stage 3 experiments such as SuperCDMS, Xenon or Zeplin4 aim to reach the $10^{-9}$ pb level. We see from Table 1 that none of the Points 1-4 will yield an observable signal at Stage 2 detectors, while just Point 2 will be detectable at a Stage 3 detector. Likewise, neutrino telescopes such as IceCube are expected to probe neutralino annihilation to SM particles in the core of the sun. The SM particles will produce high energy neutrinos, which can be detected in polar ice via conversion to muons at the level of 40 events $/ \mathrm{km}^{2} / \mathrm{yr}$, for $E_{\mu}>50 \mathrm{GeV}$. With this criterion, none of the points in the Table will be visible at IceCube.

Neutralino dark matter can also be detected via annihilations in the galactic core or halo to $\gamma \mathrm{s}, e^{+} \mathrm{s}, \bar{p} \mathrm{~s}$ or anti-deuterons $\bar{D}[28]$. We focus on gamma ray signals emanating from the galactic core since the signal is expected to be the largest in this direction. In this case, the gamma rays arise from neutralino annihilation to SM particles in the galactic core, where the SM particles hadronize to pions, and $\pi^{0} \rightarrow \gamma \gamma$. In this case, we expect a continuous signal distribution where $1 \mathrm{GeV}<E_{\gamma}<m_{\widetilde{Z}_{1}}$ (the lower limit comes from the minimum energy we require for the GLAST detector). In fact, the upper cut-off in the gamma energy distribution, if observed, would give a measure of the WIMP mass. It should, however, be kept in mind that HESS 29] and MAGIC 30] experiments have detected $\mathrm{TeV}$ gamma rays with energies ranging from their detectability threshold $\sim 200 \mathrm{GeV}$ to around $10 \mathrm{TeV}$ from the galactic center. The lower end of this range overlaps with the energies of the gamma rays expected from neutralino annihilation because, in the scenarios 
we considered here, $m_{\widetilde{Z}_{1}}$ typically ranges between $100-1000 \mathrm{GeV}$. Thus any LSP signal from the galactic center will have to be identified as an excess above this continuum, with a cut off in its energy spectrum at $m_{\widetilde{Z}_{1}}$. For LSP annihilation from regions away from the center of our Galaxy, the gamma rays detected by HESS and MAGIC experiments will not be an issue, but the signal may be smaller.

The GLAST experiment expects to be able to detect $\gamma \mathrm{s}$ at the $10^{-10}$ events $/ \mathrm{cm}^{2} / \mathrm{sec}$ level for $E_{\gamma}>1 \mathrm{GeV}$. Using this criterion, and the Adiabatically Contracted N03 Halo model[31], we find that Points 2-4 might find detectable signals from the direction of the galactic center. It should be remembered though that the gamma ray flux is sensitive to the assumed halo profile, and that our assumption of the Adiabatically Contracted N03 Halo model gives us an optimistic projection for the rate. The rates for indirect dark matter detection using an alternative less optimistic halo density profile (the Burkert profile [32]) are listed in parenthesis in the table below the rates using the N03 profile. Using the Burkert profile, none of the points would be detectable via gamma rays from the galactic center.

Turning to antimatter experiments, we compute the solar-modulated positron, antiproton and antideuteron fluxes, following the procedure outlined in Ref. [33]. We calculate the neutralino annihilation rates to $\bar{p}$ and $\bar{n}$ using the Pythia 6.154 Monte Carlo code 34 as implemented in DarkSUSY [27], and then deduce the $\bar{D}$ yield using the prescription suggested in Ref. [35]. For the propagation of charged cosmic rays through the galactic magnetic fields, we use the default DarkSUSY model where propagation is worked out through an effective two-dimensional diffusion model in the steady state approximation. We refer the reader to the DarkSUSY manual for more details 27]. Solar modulation effects are implemented through the analytical force-field approximation of Gleeson and Axford [36].

For positrons and anti-protons we evaluate the averaged differential antiparticle flux in a projected energy bin centered at a kinetic energy of $20 \mathrm{GeV}$, where we expect an optimal statistics and signal-to-background ratio at space-borne antiparticle detectors 37, 38]. We take the experimental sensitivity to be that of the Pamela experiment after three years of data-taking as our benchmark: $2 \times 10^{-9}$ events $/ \mathrm{GeV} / \mathrm{cm}^{2} / \mathrm{sec} / \mathrm{sr}$ for positrons, and $3 \times 10^{-9}$ events $/ \mathrm{GeV} / \mathrm{cm}^{2} / \mathrm{sec} / \mathrm{sr}$ for anti-protons. We find that, even with the optimistic assumption of the N03 halo profile, none of the points will yield an observable signal in the Pamela experiment via positrons or anti-protons.

Finally, the average differential antideuteron flux has been computed in the $0.1<$ $T_{\bar{D}}<0.25 \mathrm{GeV}$ range, where $T_{\bar{D}}$ stands for the antideuteron kinetic energy per nucleon, and compared to the estimated GAPS sensitivity for an ultra-long duration balloonborne experiment [39] (see Ref. [40] for an updated discussion of the role of antideuteron searches in DM indirect detection). With a projected GAPS sensitivity of $3 \times 10^{-13}$ events $/ \mathrm{GeV} / \mathrm{cm}^{2} / \mathrm{sec} / \mathrm{sr}$, just Point 2 may lead to an observable signal with the N03 halo profile, though the signal from Point 3 is right on the edge of detectability.

While the direct or indirect detection of DM may be difficult or impossible for most of the parameter range in this scenario, primarily because the LSP is bino-like, we should keep in mind that there are regions where agreement with (1.2) may be obtained with parameters in the $A$-funnel 28]; see Fig. 8. In this case, we may expect that detection becomes possible 
via direct detection experiments, and via indirect anti-particle and gamma ray searches, but not in IceCube (because the spin-dependent LSP nucleon cross section is not enhanced).

\section{MM-AMSB Model with non-zero modular weights}

The results of the previous section were all obtained in MM-AMSB model where the modular weights $n_{i}$ were all taken to be zero. Many other choices of modular weights may be taken depending on which branes the matter and Higgs fields inhabit. Each choice will yield a somewhat different low energy phenomenology, since the soft terms all depend on the modular weights. In this section, we will illustrate how the phenomenology changes for a particular choice: we will retain $\ell_{a}=1$, but take Higgs fields to live in a $D 3$ brane, so that $n_{H_{u}}=n_{H_{d}}=1$, while matter fields live on intersections of $D 7$ branes, so that $n_{\text {matter }}=\frac{1}{2}$. We will, for brevity, refer to this as the non-zero modular weights (NZMW) model. We have also examined the choice $n_{\text {matter }}=1, n_{H_{u}}=n_{H_{d}}=0$. We will discuss this case briefly at the end of Section 4.2 .

\subsection{Soft SUSY breaking terms}

While the gaugino SSB mass parameters are unaltered, the soft SUSY breaking terms for the scalars are modified from the values presented in Fig. 1. In general, a non-zero choice for the modular weights reduces importance of the modulus-mediated contributions to the SSB masses and $A$-parameters relative to the AMSB contributions. For our particular choice $n_{H_{u}}=n_{H_{d}}=1$ and $n_{\text {matter }}=\frac{1}{2}$, the $a_{i j k}$ coefficients of the $\alpha$ term in Eq. 2.7 are diminished, so that the common GUT scale value of the $A$-parameters is now $\sim-M_{s}$ rather than $-3 M_{s}$, and these no longer dominate $X_{t}$ as in the previous section. In addition, the $c_{i}$ coefficients in Eq. 2.8 are diminished, which enhanced the AMSB and mixed modulusanomaly mediated contributions to the soft scalar squared masses.

As an example, we plot in Fig. 10 the same soft parameters as in Fig. 1, but for the NZMW case. In frame $a$ ), the gaugino masses are of course unaffected. However, the GUT scale values of trilinear $A$-parameters are substantially reduced in magnitude compared to Fig. 1 1 a). Thus, we expect in the case of the NZMW model a reduced diminution of the top squark soft terms, and hence a heavier $\tilde{t}_{1}$. In frame $b$ ), we see the third generation and Higgs scalar masses. Taking $n_{H_{u}}=n_{H_{d}}=1$ means $c_{H_{u}}=c_{H_{d}}=0$, so that the pure modulusmediated contribution to Higgs squared masses is absent. In fact, the Higgs squared masses have negative values for the entire range of $\alpha$ shown. In addition, the modulus-mediated contribution to the squark and slepton soft masses is diminished compared to the zero modular weights case. This results in the mixed anomaly/modulus mediation contribution dominating the soft masses for a larger range of $\alpha$ than in the case of zero modular weights, so that GUT scale soft squared masses are also negative over a range of $\alpha$ values. As before, after renormalization group evolution to low scales, we see that over a portion of this range of $\alpha$ we find acceptable spectra at the weak scale.

In Fig. 11 $a$ ) we show the evolution of gaugino masses for the case $\alpha=6, m_{3 / 2}=11.5$ $\mathrm{TeV}, \tan \beta=10$ and $\mu>0$. They evolve in essentially the same fashion as Fig. 2 (since the effects of modular weights only enter either via decoupling in the RGEs, or via two 
loop terms in the RGE), and again show mirage unification at $Q \sim 10^{11} \mathrm{GeV}$. The $A_{t}$, $A_{b}$ and $A_{\tau}$ evolution is shown in frame $b$ ). In this case, these parameters evolve to weak scale values which are comparable to the other soft masses. While they do not show exact mirage unification owing to Yukawa coupling effects in their evolution, these apparently unify much better than in the case of the ZMW model because the Yukawa coupling terms in the evolution of the $A$-parameters are now smaller because of the reduced values of the A-parameters.

In Fig. 12, we show the evolution of scalar SSB mass parameters from $Q=M_{G U T}$ to $Q=M_{\text {weak }}$, for the same model choice as in Fig. 11. In this case, it is interesting to

note that $m_{H_{u}}^{2}$ first evolves from negative to positive values, and then back to negative again, i.e it would have been premature to conclude that electroweak symmetry is broken at the tree-level from the fact that the Higgs boson squared mass parameters were negative. We also see that though several matter SSB mass squared parameters are negative at the GUT scale, they ultimately evolve to positive values, and we obtain acceptable weak scale spectra. This re-iterates comments made in Sec. 3 about the importance of the radiative corrections to the potential with parameters renormalized at a scale much higher than the weak scale. Note that the matter scalars and the Higgs scalars now show separate (approximate) mirage unification at the common scale of $Q \sim 10^{11} \mathrm{GeV}$.

\subsection{Mass spectrum}

In Fig. 13, we plot the physical sparticle masses versus $\alpha$ for $m_{3 / 2}=11.5 \mathrm{TeV}, \mu>0$ and $a) \tan \beta=10$ and $b) \tan \beta=30$. In both cases, $|\mu|$ is large over most of the range of $\alpha$, resulting in a bino-like LSP. Also, in both cases, the $\tilde{\tau}_{1}$ is the NLSP, and in fact the lower range of (positive) $\alpha$ is bounded by the requirement of an uncharged LSP. A striking feature is the narrow allowed sliver of $\alpha>0$ between the red and blue regions in frame $b$ ). In this region, $\mu$ also becomes very small, and mixing between the bino and the higgsino depresses the LSP mass below $m_{\tilde{\tau}_{1}}$, and the LSP is a roughly equal mixture of bino, wino and the higgsinos. We also see that the $\tilde{t}_{1}$ is seen to be typically much more massive than the $\widetilde{Z}_{1}$, so that in this case top squark co-annihilation plays no role in reducing the relic density. However, there does exist a range of $\alpha$ values for which $2 m_{\widetilde{Z}_{1}} \sim m_{A}(\alpha \sim 6-8$ in frame $a$ ) and $\alpha \sim 8-11$ in frame $b$ )), so that $A$-funnel annihilation can act to reduce the relic density over some range of parameter choices.

In Fig. 14, we plot the physical sparticle masses versus $\tan \beta$ for $\alpha=6, m_{3 / 2}=11.5$ $\mathrm{TeV}$, and $a) \mu>0$ and $b) \mu<0$. In this case, again the magnitude of $\mu$ stays large, while the upper bound on $\tan \beta$ comes from the requirement of a neutralino LSP. In both frames, we see that there is a range a $\tan \beta$ where $2 m_{\widetilde{Z}_{1}} \sim m_{A}$, so that there is efficient resonant annihilation of relic neutralinos. Notice that $m_{\widetilde{W}_{1}}: m_{\widetilde{Z}_{1}}$ differs significantly from $2: 1$ expected in models with gaugino mass unification.

The relic density for the NZMW model for the same parameters as in Fig. 14 is shown in Fig. 15. In both frames stau co-annihilation reduce the relic density at the upper end of the range of $\tan \beta$, while at the lower value of $\tan \beta$, this reduction occurs via $s$-channel resonant annihilation via $A, H$ bosons. 
In Fig. 16, we plot the allowed regions of the MM-AMSB model with non-zero modular weights in the $\alpha$ vs. $m_{3 / 2}$ plane for $\mu>0$ and $\left.a\right) \tan \beta=10$ and $\left.b\right) \tan \beta=30$. The notation used is the same as in Fig. 9. Aside from the white region where either the theoretical constraints or the bounds from LEP 2 searches are not satisfied, portions of the plane are excluded when the $\tilde{\tau}_{1}$ becomes the LSP. This is in contrast to the ZMW model where large parts of this plane were excluded because $\tilde{t}_{1}$ becomes the LSP. The boundary between the green $\times$ and red + regions gives $\Omega_{\widetilde{Z}_{1}} h^{2} \sim 0.11$. For positive values of $\alpha$, both frames are qualitatively similar: for large $m_{3 / 2}$ with $\alpha \sim 5$ or 6 , the WMAP allowed region occurs due to stau co-annihilation, and extends to $m_{3 / 2}>60 \mathrm{TeV}$. In both frames, to the left of the stau LSP region we have a portion of the plane where the relic density is reduced to below that in (1.2). In this region $M_{1}, M_{2}$ and $\mu$ are comparable and the LSP is higgsino-like but has significant bino and wino components. This would facilitate the observation of direct and indirect LSP signals, but the signal size would depend on what fraction of DM density is composed of neutralinos. In both frames there is a peak structure around $\alpha \sim 6-8$ (frame $a$ ) or $\alpha \sim 10$ (frame $b$ ) where $2 m_{\widetilde{Z}_{1}} \sim m_{A}$ and neutralino annihilation can occur via the $A$-funnel. For larger values of $\alpha$, very low values of $m_{3 / 2}$ are required to obtain consistency with (1.2), which can then only occur via neutralino annihilation through low mass $t$-channel sfermion exchange (bulk annihilation).

Turning to negative values of $\alpha$, in frame $a$ ) we see a region of red points close to $\alpha \sim-1.5$. Near the boundary between the red and blue points, the neutralino relic density saturates the observed CDM relic density via BWCA, while in bulk of the region with the red points, the annihilation is too rapid and leads to a smaller density of relic neutralinos. As for the model with zero modular weights, we do not have a corresponding region in frame $b$ ).

We illustrate sample spectra in the MM-AMSB model with non-zero modular weights with the four points listed in Table 2. The first case, labelled Point 5 , has $m_{3 / 2}=40 \mathrm{TeV}$ and $\tan \beta=10$, just as Point 1 of Table 1, but with $\alpha=4.7$. It is a stau co-annihilation point. Point 6 is taken at $\alpha=8, m_{3 / 2}=25.467 \mathrm{TeV}$ so that it lies in the $A$-annihilation funnel. Sparticles and non-SM Higgs bosons are relatively heavy for both these points and give only small contributions to the $B F(b \rightarrow s \gamma)$ and to the anomalous magnetic moment. Point 7 illustrates a spectrum for $\tan \beta=30$ with $\alpha=8, m_{3 / 2}=10.5 \mathrm{TeV}$, and is located at the intersection of the stau co-annihilation and $A$-funnel regions. It has gluino and squark masses around $1 \mathrm{TeV}$, while sleptons and the lighter inos have masses that are accessible at a $1 \mathrm{TeV} e^{+} e^{-}$collider. The branching fraction for $b \rightarrow s \gamma$ is on the low side of its acceptable range and there is a modest SUSY contribution to the muon $g-2$. We see that the $B F\left(B_{s} \rightarrow \mu^{+} \mu^{-}\right)$deviates considerably from its SM value, primarily because of the larger value of $\tan \beta$. Finally, we choose Point 8 with a negative value of $\alpha=-1.64$, with $m_{3 / 2}=24 \mathrm{TeV}$ and $\tan \beta=10$ where BWCA yields to a neutralino relic density in accord with (1.2). We have checked that for $\alpha$ close to this value, the relic neutralino density saturates the measured CDM density over the entire range of $m_{3 / 2}$ where we have red $+\mathrm{s}$ in Fig. 16: less negative values of $\alpha$ yield a lighter chargino and the neutralino relic density becomes too low, so that another source of DM is also needed in this region. For this case, squark and gluino masses are similar to those for Point 7, but sleptons, and especially 


\begin{tabular}{|c|c|c|c|c|}
\hline parameter & Point 5 & Point 6 & Point 7 & Point 8 \\
\hline$\alpha$ & 4.7 & 8 & 8 & -1.64 \\
\hline$m_{3 / 2}(\mathrm{TeV})$ & 40 & 25.467 & 10.5 & 24 \\
\hline $\tan \beta$ & 10 & 10 & 30 & 10 \\
\hline$\mu$ & 1022.9 & 1425.2 & 625.6 & 816.4 \\
\hline$m_{\tilde{g}}$ & 1808.8 & 2304.6 & 1010.6 & 1112.5 \\
\hline$m_{\tilde{u}_{L}}$ & 1644.1 & 2158.8 & 948.2 & 1073.9 \\
\hline$m_{\tilde{t}_{1}}$ & 1254.5 & 1601.5 & 679.6 & 765.6 \\
\hline$m_{\tilde{b}_{1}}$ & 1520.5 & 1971.1 & 832.0 & 953.4 \\
\hline$m_{\tilde{e}_{L}}$ & 985.7 & 1149.0 & 479.9 & 308.7 \\
\hline$m_{\tilde{e}_{R}}$ & 886.3 & 993.0 & 412.2 & 199.0 \\
\hline$m_{\tilde{\tau}_{1}}$ & 879.8 & 982.9 & 352.2 & 185.2 \\
\hline$m_{\widetilde{W}_{1}}$ & 990.6 & 1110.5 & 441.1 & 135.0 \\
\hline$m_{\widetilde{Z}_{2}}$ & 990.6 & 1108.9 & 440.6 & 134.4 \\
\hline$m_{\widetilde{Z}_{1}}$ & 869.1 & 791.2 & 315.0 & 114.1 \\
\hline$m_{A}$ & 1135.2 & 1581.2 & 595.8 & 829.4 \\
\hline$m_{h}$ & 119.7 & 120.8 & 116.7 & 114.9 \\
\hline$\Omega_{\widetilde{Z}_{1}} h^{2}$ & 0.12 & 0.12 & 0.11 & 0.10 \\
\hline$B F(b \rightarrow s \gamma)$ & $3.3 \times 10^{-4}$ & $3.4 \times 10^{-4}$ & $2.4 \times 10^{-4}$ & $4.1 \times 10^{-4}$ \\
\hline$B F\left(B_{s} \rightarrow \mu^{+} \mu^{-}\right)$ & $3.9 \times 10^{-9}$ & $3.9 \times 10^{-9}$ & $6.3 \times 10^{-9}$ & $3.8 \times 10^{-9}$ \\
\hline$\Delta a_{\mu}$ & $1.3 \times 10^{-10}$ & $9.4 \times 10^{-11}$ & $16.2 \times 10^{-10}$ & $-1.0 \times 10^{-10}$ \\
\hline$\sigma_{s c}\left(\widetilde{Z}_{1} p\right)$ & $4.4 \times 10^{-9} \mathrm{pb}$ & $1.3 \times 10^{-10} \mathrm{pb}$ & $2.1 \times 10^{-9} \mathrm{pb}$ & $7.8 \times 10^{-11} \mathrm{pb}$ \\
\hline$\Phi^{\mu}\left(k m^{-2} y r^{-1}\right)$ & 1.43 & 0.03 & 0.45 & 0.002 \\
\hline$\Phi^{\gamma}\left(c m^{-2} s^{-1}\right)$ & $\begin{array}{c}1.6 \times 10^{-9} \\
\left(8.3 \times 10^{-14}\right)\end{array}$ & $\begin{array}{c}5.2 \times 10^{-7} \\
\left(2.6 \times 10^{-11}\right)\end{array}$ & $\begin{array}{c}2.5 \times 10^{-7} \\
\left(1.2 \times 10^{-11}\right)\end{array}$ & $\begin{array}{l}9.2 \times 10^{-10} \\
\left(4.7 \times 10^{-14}\right)\end{array}$ \\
\hline$\Phi^{e^{+}}\left(\mathrm{GeV}^{-1} \mathrm{~cm}^{-2} s^{-1} s r^{-1}\right)$ & $\begin{array}{l}4.9 \times 10^{-11} \\
\left(9.6 \times 10^{-12}\right)\end{array}$ & $\begin{array}{l}1.8 \times 10^{-8} \\
\left(3.7 \times 10^{-9}\right)\end{array}$ & $\begin{array}{l}3.7 \times 10^{-9} \\
\left(8.7 \times 10^{-10}\right)\end{array}$ & $\begin{array}{l}8.3 \times 10^{-11} \\
\left(1.9 \times 10^{-11}\right)\end{array}$ \\
\hline$\Phi^{\bar{p}}\left(G e V^{-1} c^{-2} s^{-1} s r^{-1}\right)$ & $\begin{array}{l}2.4 \times 10^{-10} \\
\left(1.4 \times 10^{-11}\right)\end{array}$ & $\begin{array}{l}7.7 \times 10^{-8} \\
\left(4.4 \times 10^{-9}\right)\end{array}$ & $\begin{array}{l}3.2 \times 10^{-8} \\
\left(1.8 \times 10^{-9}\right)\end{array}$ & $\begin{array}{l}1.0 \times 10^{-11} \\
\left(5.8 \times 10^{-13}\right)\end{array}$ \\
\hline$\Phi^{\bar{D}}\left(G e V^{-1} c m^{-2} s^{-1} s r^{-1}\right)$ & $\begin{array}{l}5.0 \times 10^{-14} \\
\left(4.2 \times 10^{-15}\right)\end{array}$ & $\begin{array}{l}2.4 \times 10^{-11} \\
\left(2.1 \times 10^{-12}\right)\end{array}$ & $\begin{array}{l}1.6 \times 10^{-11} \\
\left(1.4 \times 10^{-12}\right)\end{array}$ & $\left(3.5 \times 10^{-15}\right)$ \\
\hline
\end{tabular}

Table 2: Masses and parameters in GeV units for case studies 5-8 within the NZMW framework with $n_{H_{u}}=n_{H_{d}}=1$, and $n_{\text {matter }}=1 / 2$. Also shown are predictions for low energy observables along with cross sections and fluxes relevant to direct and indirect searches for dark matter. In all cases, we take $m_{t}=175 \mathrm{GeV}$ and $\mu>0$. The halo annihilation rates use the Adiabatically Contracted N03 Halo model, while the values in parenthesis use the Burkert halo profile.

the lighter charginos and neutralinos are considerably lighter. As for the model with zero modulus weights, the contribution to muon $g-2$ is small and negative, while $B F(b \rightarrow s \gamma)$ is close to the upper end of its acceptable range.

Before turning to the discussion of collider signals, we note that by choosing different values for the modular weights for matter and Higgs supermultiplets we can obtain the so-called non-universal Higgs mass (NUHM) model[41], where the GUT scale values of $m_{H_{u}}^{2}$ and $m_{H_{d}}^{2}$ are split off from the masses of the matter scalars. Moreover, if the modular weights for the Higgs multiplets are chosen to be the same but larger than those for matter 
multiplets, the (common) GUT scale Higgs boson mass squared parameter, $m_{\phi}^{2}$, is (much) larger than the corresponding matter parameters, and we get what has been referred to as the NUHM1 model with large $m_{\phi}^{2} / m_{0}^{2} \cdot{ }^{9}$ It is then possible for $|\mu|$ to become small enough so that the neutralino is MHDM 41. Motivated by these considerations, we examined the case, $n_{H_{u}}=n_{H_{d}}=0, n_{\text {matter }}=1$. In Fig. 17, we show the results of our scan of the $\alpha-m_{3 / 2}$ parameter plane for this choice of modular weights, with $\tan \beta=10$. We see that most of the region of large positive $\alpha$ where we may have expected to obtain MHDM is excluded because the stau becomes lighter than $\widetilde{Z}_{1}$. This situation may be different if we take $n_{\text {matter }}=1 / 2$, but then the ratio $m_{\phi}^{2} / m_{0}^{2}$ is also reduced; we have not examined this possibility. For negative values of $\alpha$, we see that there is a viable region with red points near $\alpha \simeq-2$, just a bit below where we had obtained the BWCA solution in the previous figure. We have checked that agreement with (1.2) is obtained via stau co-annihilation, and that the difference $\left|M_{2}\right|-\left|M_{1}\right|$ is indeed too large for BWCA. Consistency with the relic density constraint also obtains for very small values of $m_{3 / 2}$ via bulk annihilation: these points, however, have a rather large negative SUSY contribution to $(g-2)_{\mu}$, and so are strongly disfavored. Finally, we note that unlike previous cases, for this choice of modular weights a TeV LC will be able to probe ranges of parameters beyond the LHC. This is primarily because $\tilde{\tau}_{1}$, and to a smaller extent, also $\tilde{e}_{R}$ and $\tilde{\mu}_{R}$ are accessible even for $m_{\tilde{g}} \simeq m_{\tilde{q}} \gtrsim 3 \mathrm{TeV}$.

\subsection{Prospects for collider and dark matter search experiments}

As in the case of the MM-AMSB framework with zero modular weights, the NZMW model generally produces SUSY spectra with $m_{\tilde{q}} \sim m_{\tilde{g}}$. This is because unless $|\alpha|$ is very small, the GUT scale gluino mass parameters are comparable to (or larger than) the corresponding squark parameters, so that bulk of the physical squark and gluino masses come from the renormalization group evolution to the weak scale. This is analogous to the more familiar situation in mSUGRA when $m_{0} \lesssim m_{1 / 2}$. Since, as we discussed in the last section, the reach of the LHC extends out to $m_{\tilde{g}} \sim m_{\tilde{q}} \simeq 3 \mathrm{TeV}$ for this case, we expect a similar reach for the NZMW model, assuming an integrated luminosity of $100 \mathrm{fb}^{-1}$. This reach is shown by the red contours in Fig. 16, which generally track the $m_{\tilde{g}} \sim 3 \mathrm{TeV}$ contour, except when they are on the edge of the allowed parameter space, as for instance for $\alpha<0$ in frame a). It is noteworthy that the LHC reach generally encompasses the entire $A$-annihilation funnel. In fact, only a small stau co-annihilation region with $m_{3 / 2}>60 \mathrm{TeV}$ and $\alpha \sim 5.5$ at low $\tan \beta$ can escape LHC detection. The LHC with $100 \mathrm{fb}^{-1}$ can cover all the relicdensity-allowed parameter space for $\tan \beta=30$. The reach of a linear $e^{+} e^{-}$collider is mainly determined by the kinematic reaches for chargino and $\tilde{\tau}_{1}$ pair production. A 500 $\mathrm{GeV}$ collider covers only a small portion of the allowed parameter plane, and even at a $1 \mathrm{TeV}$ LC considerable portions of the stau co-annihilation region will not be covered. It is, however, interesting that the entire BWCA region in frame $a$ ) can be probed at a LC, since it is only via LC experiments that we will have any chance of directly probing the small mass difference between the chargino and the LSP.

\footnotetext{
${ }^{9}$ More precisely, it is the NUHM1 model except for the splitting between $m_{H_{u}}^{2}$ and $m_{H_{d}}^{2}$ due to the AMSB contribution.
} 
Points 5 and 6 should be observable via multijet + multilepton $+E_{T}^{\text {miss }}$ signatures at the LHC, although their spectra, other than the light Higgs, will be inaccessible to either of the LC options. In both cases, gluinos decay a significant fraction of the time to third generation squarks, so that LHC SUSY events should be rich in $b$-jets. It may also be possible to access the very heavy $\widetilde{W}_{1}$ and $\widetilde{Z}_{2}$ (and for Point 5 , also $\widetilde{Z}_{4}$ and $\widetilde{W}_{2}$ ) via cascade decays of $\tilde{q}_{L}$. Point 7 should easily be visible at LHC. Once again, gluinos decay preferentially to the third generation. Decays of these and of the light squarks yield $\widetilde{W}_{1}$ and $\widetilde{Z}_{2}$ at large rates. The latter mostly decays via $\widetilde{Z}_{2} \rightarrow \tilde{\tau}_{1} \tau$ and $\widetilde{Z}_{2} \rightarrow h \widetilde{Z}_{1}$ so that construction of mass edges may be difficult: see, however Ref.42]. Although sparticles are essentially inaccessible at a $500 \mathrm{GeV} \mathrm{LC}$, a $1 \mathrm{TeV}$ collider will provide access to sleptons and light chargino/neutralino pairs, though some of the rates may be suppressed by phase space. Finally, at Point 8, there should again be a plethora of signals at the LHC. Sparticle decay patterns are qualitatively similar to those for Point 7 , but the big difference is the approximate equality: $m_{\widetilde{Z}_{1}} \simeq m_{\widetilde{W}_{1}} \simeq m_{\widetilde{Z}_{2}}$. As a result, the decay products of $\widetilde{W}_{1}$ and $\widetilde{Z}_{2}$ will be relatively soft at the LHC. In this case, though, $\tilde{\tau}_{1}, \tilde{e}_{R}$ and the lighter charginos and neutralinos should be accessible at even a $500 \mathrm{GeV}$ LC, whereas all sleptons are accessible at $1 \mathrm{TeV}$. As mentioned above, LC experiments may be crucial if the BWCA mechanism is what reduces the relic density.

Turning to dark matter searches in the NZMW scenario, we see that none of the Points 5-8 will lead to an observable signal at CDMS2, though Points 5 and 7 may be observable at a stage 3 detector such as a 1 ton Xenon facility. The sensitivity of IceCube is too low for all these points. Prospects for detection of gamma rays or anti-particles from neutralino annihilation appear to be better in the NZMW case relative to the case of zero modular weights. For Point 5 (relative to Point 1 which has a quite similar spectrum) this is because of the increased higgsino component in the LSP. Point 6 (7) is in (on the edge of) the $A$ funnel, so that an increased rate should not be a surprise. Assuming the same sensitivity as in the last section, we see that there should be a gamma ray signal in GLAST for all the points using the N03 halo profile, while none of the points are observable using the Burkert profile. Pamela should have observable positron and anti-proton signals for Points 6 and 7 (Point 6) in the N03 profile (Burkert profile). Points 6 and 7 also give a detectable anti-deuteron signal for both halo profiles.

\section{Summary and Conclusions}

The illustration by Kachru et al. [1] that compactifications with fluxes in extra spatial dimensions in type IIB string models can stabilize the moduli and give rise to a de Sitter vacuum for the Universe has spurred several recent studies of the structure of the soft SUSY breaking terms in these scenarios. The structure of these terms depend on certain integers or half integers, the so-called modular weights of the MSSM superfields, that characterize their location in the extra dimensions. Phenomenologically, the most important feature of this scenario is that the SSB parameters can obtain comparable contributions from the mediation of SUSY breaking by moduli fields and the so-called anomaly mediation of SUSY breaking, in contrast to previously studied models where anomaly-mediated SUSY 
breaking effects were considered to be negligible unless mediation by moduli is, for one reason or other, essentially negligible. The relative strength of modulus and anomalymediated contributions is controlled by a phenomenological parameter $\alpha$ which can assume any (positive or negative) real number. The value of $\alpha$ (and many other parameters) will be fixed if it ever becomes possible to explicitly construct a realistic vacuum starting from string theory. Until this time, we have to use this construction solely as a motivation for the examination of the phenomenology of MM-AMSB models.

Flavor changing neutral current constraints suggest that there should not be large mass splittings between sparticles with the same gauge quantum numbers. This can be ensured by choosing common modular weights for super-multiplets with the same gauge quantum numbers. This consideration, by itself, does not constrain the modular weights of different MSSM multiplets, but of course, if we want to embed these in a GUT, we should choose a common modular weight for all particles in the same GUT multiplet. Viewed differently, these should all be at the same location in the extra dimension. Throughout the paper, we assume that all gauginos also reside at a common location, and so receive a common mass from modulus mediation.

We have begun our phenomenological study assuming, for simplicity, that the modular weights are all zero. This gives a universal modulus-mediated contribution to all GUT scale scalar mass parameters, to the trilinear $A$-parameters and to the gaugino masses. Moreover, the GUT scale values of these contributions are in the ratio, $m_{0}: m_{1 / 2}: A_{0}=1: 1:-3$. Of course, the usual anomaly-mediated contribution has also to be included, and for scalar mass parameters, there is a mixed modulus-anomaly-mediated contribution also. The GUT scale values of these SSB parameters are given by (2.6)-(2.7). The framework is more general than the well-studied mSUGRA or AMSB frameworks in that $\alpha$ allows us to control the relative strengths of the respective contributions, but is more constrained in that the universal contributions to all SSB parameters are in a fixed ratio. Indeed, once the modular weights are fixed, the parameter space is smaller than that of the mSUGRA model.

Turning to the phenomenology of the model with zero modular weights, we find that the spectrum is characterized by a relatively light $t$-squark or tau slepton. This is because of the large value of the $A$-parameter. Indeed, for positive values of $\alpha$, consistency with the observed relic density is usually obtained only when the LSP, which is mostly bino-like, can co-annihilate with either $\tilde{t}_{1}$ or $\tilde{\tau}_{1}$ : Higgs funnel annihilation is possible for a limited range of parameters. Because the ratio $M_{1}: M_{2}$ can be adjusted by an appropriate choice of $\alpha$, it may appear that it should be possible to adjust it to obtain MWDM (by setting $M_{1}($ weak $) \simeq M_{2}($ weak $\left.)\right)$ or BWCA (by setting $M_{1}$ (weak $) \simeq-M_{2}$ (weak)). We found, however, that MWDM is not possible because the $\tilde{t}_{1}$ becomes the LSP for the required value of $\alpha$. It is, however, possible to obtain agreement with the relic density constraint via BWCA by choosing $\alpha \sim-1.7$. Experiments at the LHC will essentially probe the entire range of parameters of the MM-AMSB model with zero modular weights for parameters consistent with the determination of the DM relic density. However, because the LSP is mostly a bino that co-annihilates with the stop or the stau, signals for direct and indirect detection of neutralino DM are usually small. 
We also presented results for one case of non-zero modular weights, with $n_{H_{u}}=n_{H_{d}}=$ 1 and $n_{\text {matter }}=\frac{1}{2}$. In this case, the top squark mass is much heavier than in the ZMW case. However, WMAP allowed regions can still be found via either stau co-annihilation, $A$-funnel annihilation, mixed higgsino/wino DM or via BWCA. The CERN LHC can cover almost all of the interesting parameter space. A combination of LHC and LC measurements may allow the SSB parameters to be extracted: their extrapolation to higher scales via RGE should exhibit "mirage unification", as do the ZMW solutions. Prospects for DM detection are somewhat better for this choice of modular weights, primarily because $A$ funnel annihilation is possible for some ranges of model parameters.

In summary, we have explored the phenomenology of a novel MM-AMSB framework where MSSM SSB parameters receive comparable modulus- and anomaly-mediated SUSY breaking contributions. The framework leads to unusual sparticle mass patterns, and can naturally accommodate the BWCA scenario. Experiments at the LHC will be able to explore almost all parameter regions consistent with the measured CDM density, while only limited ranges of the parameter space will be available to LC experiments. Fortunately, experiments at a LC will be able to perform detailed studies of charginos and neutralinos if nature chooses the BWCA mechanism to make the relic density in accord with observation. These studies are difficult at the LHC on account of the small mass gaps between $\widetilde{Z}_{1}, \widetilde{Z}_{2}$ and $\widetilde{W}_{1}$. In the case that the soft terms are able to be extracted via a combination of LHC and LC measurements, then the phenomenon of "mirage unification" should be evident, especially for the gaugino masses.

\section{Acknowledgments}

We thank K. Choi, A. Falkowski and Y. Mambrini for helpful correspondence. This research was supported in part by the U.S. Department of Energy grants DE-FG02-97ER41022 and DE-FG03-94ER40833.

\section{NOTE ADDED}

The phenomenology of the model with NZMW was also examined by Kitano and Nomura 433. In their analysis, they fix the mirage unification scale to be $\sim \mathrm{TeV}$ which, in turn, implies $\alpha \simeq 3$, corresponding to the small $\mu$ solutions that we find in Fig. 16. However, while we obtain $m_{A}$ and $\mu$ via two loop renormalization group evolution using the boundary values of $m_{H_{u}}^{2}$ and $m_{H_{d}}^{2}$ as given in (2.8), Kitano and Nomura, who essentially perform a one-loop analysis, argue that two loop renormalization group effects would generate nonzero values of the Higgs boson squared mass parameters at the mirage unification scale, and so trade these for $\mu$ and $m_{A}$ which they treat as free parameters that they vary in the range $|\mu|<190 \mathrm{GeV}$ and $m_{A}<300 \mathrm{GeV}$, where the range follows from the requirement that their naturalness parameter $\Delta<0.2$. This same requirement bounds the gravitino mass scale giving them a light sparticle spectrum, corresponding to the small $\alpha>0$ slice of the plane of Fig. 16. We see, however, from Fig. 12 that while two loop effects do indeed make $m_{H_{u}}^{2}$ and $m_{H_{d}}^{2}$ non-zero (and negative) at the mirage unfication scale, they evolve 
to a specific value, so that the values of $|\mu|$ and $m_{A}$ are completely calculable within this framework. Finally, we note that the origin of mirage unification for the NZMW model (in fact in all models where $n_{i}+n_{j}+n_{k}=2$, where $i, j$ and $k$ are the fields that enter the Yukawa couplings and trilinear soft terms; e.g. the model illustrated in Fig. 17) at the one loop level can be understood from the formulae given by Choi et al. [4], as well as from the analysis in the Appendix of the second paper in Ref.43]. We thank R. Kitano and Y. Nomura for bringing their work on the MM-AMSB model to our attention.

\section{References}

[1] S. Kachru, R. Kallosh, A. Linde and S. P. Trivedi, Phys. Rev. D 68 (2003) 046005.

[2] K. Choi, A. Falkowski, H. P. Nilles, M. Olechowski and S. Pokorski, J. High Energy Phys. 0411 (2004) 076; K. Choi, A. Falkowski, H. P. Nilles and M. Olechowski, Nucl. Phys. B 718 (2005) 113 .

[3] L. Randall and R. Sundrum, Nucl. Phys. B 557 (1999) 79; G. F. Giudice, M. Luty, H. Murayama and R. Rattazzi, J. High Energy Phys. 9812 (1998) 027; J. Bagger, T. Moroi and E. Poppitz, J. High Energy Phys. 0004 (2000) 009; P. Binetruym M. K. Gaillard and B. Nelson, Nucl. Phys. B 604 (2001) 32.

[4] K. Choi, K-S. Jeong and K. Okumura, J. High Energy Phys. 0509 (2005) 039.

[5] A. Falkowski, O. Lebedev and Y. Mambrini, J. High Energy Phys. 0511 (2005) 034.

[6] M. Endo, M. Yamaguchi and K. Yoshioka, Phys. Rev. D 72 (2005) 015004.

[7] P. G. Mercadante, J. K. Mizukoshi and X. Tata, Phys. Rev. D 72 (2005) 035009.

[8] H. Baer, C. H. Chen, M. Drees, F. Paige and X. Tata, Phys. Rev. Lett. 79 (1997) 986 and Phys. Rev. D 58 (1998) 075008.

[9] D. N. Spergel et al., astro-ph/0603449 (2006); see D. N. Spergel et al., astro-ph/0302209 (2003) for results from the analysis of the first year of WMAP data.

[10] J. Ellis, K. Olive, Y. Santoso and V. Spanos, Phys. Lett. B 565 (2003) 176; H. Baer and C. Balazs, JCAP 05 (2003) 006; U. Chattapadhyay, A. Corsetti and P. Nath, Phys. Rev. D 68 (2003) 035005; A. Lahanas and D. V. Nanopoulos, Phys. Lett. B 568 (2003) 55; for a review, see A. Lahanas, N. Mavromatos and D. Nanopoulos, Int. J. Mod. Phys. D 12 (2003) 1529.

[11] H. Baer, A. Belyaev, T. Krupovnickas and J. O'Farrill, JCAP 0408 (2004) 005.

[12] K. Choi, K. S. Jeong, T. Kobayashi and K. Okumura, Phys. Lett. B 633 (2006) 355; R. Kitano and Y. Nomura, Phys. Lett. B 631 (2005) 58; O. Lebedev, H. P. Nilles and M. Ratz, hep-ph/0511320, (2005); see also A. Pierce and J. Thaler, hep-ph/0604192 (2006).

[13] G. L. Kane, J. D. Lykken, S. Mrenna, B. D. Nelson, L. T. Wang and T. T. Wang, Phys. Rev. D 67 (2003) 045008.

[14] H. Baer, A. Mustafayev, S. Profumo, A. Belyaev and X. Tata, Phys. Rev. D 71 (2005) 095008 and J. High Energy Phys. 0507 (2005) 065.

[15] J. Feng, A. Rajaraman and B. Smith, hep-ph/0512172 (2005).

[16] H. Baer, A. Mustafayev, E. Park and S. Profumo, J. High Energy Phys. 0507 (2005) 046. 
[17] H. Baer, T. Krupovnickas, A. Mustafayev, E. Park, S. Profumo and X. Tata, J. High Energy Phys. 0512 (2005) 011.

[18] ISAJET v7.74, by H. Baer, F. Paige, S. Protopopescu and X. Tata, hep-ph/0312045; see also H. Baer, J. Ferrandis, S. Kraml and W. Porod, Phys. Rev. D 73 (2006) 015010.

[19] G. Belanger, F. Boudjema, A. Cottrant, A. Pukhov and A. Semenov, Nucl. Phys. B 706 (2005) 411 .

[20] Y. Mambrini and E. Nezri, hep-ph/0507263 (2005).

[21] H. Baer, A. Mustafayev, E. Park, S. Profumo and X. Tata, J. High Energy Phys. 0604 (2006) 041.

[22] M. Carena, M. Quiros, M. Seco and C. Wagner, Nucl. Phys. B 650 (2003) 24; C. Balazs, M. Carena and C. Wagner, Phys. Rev. D 70 (2004) 015007; for a review, see M. Trodden, hep-ph/0411301 (2004).

[23] H. Baer, C. H. Chen, F. Paige and X. Tata, Phys. Rev. D 52 (1995) 2746 and Phys. Rev. D 53 (1996) 6241; H. Baer, C. H. Chen, M. Drees, F. Paige and X. Tata, Phys. Rev. D 59 (1999) 055014; S. Abdullin and F. Charles, Nucl. Phys. B 547 (1999) 60; S. Abdullin et al. (CMS Collaboration), hep-ph/9806366; B. Allanach, J. Hetherington, A. Parker and B. Webber, J. High Energy Phys. 08 (2000) 017; for a recent update, see H. Baer, C. Balazs, A. Belyaev, T. Krupovnickas and X. Tata, J. High Energy Phys. 0306 (2003) 054.

[24] H. Baer, J. Sender and X. Tata, hep-ph/9308376 and Phys. Rev. D 50 (1994) 4517; R. Demina, J. Lykken, K. Matchev and A. Nomerotski, Phys. Rev. D 62 (2000) 035011.

[25] H. Baer, A. Belyaev, T. Krupovnickas and X. Tata, J. High Energy Phys. 0402 (2004) 007;

H. Baer, T. Krupovnickas and X. Tata, J. High Energy Phys. 0406 (2004) 061.

[26] The Isatools neutralino-nucleon scattering rate is discussed in H. Baer, C. Balazs, A. Belyaev and J. O'Farrill, JCAP 0309 (2003) 007.

[27] P. Gondolo, J. Edsjo, P. Ullio, L. Bergstrom, M. Schelke and E. A. Baltz, JCAP 0407 (2004) 008 [arXiv:astro-ph/0406204].

[28] H. Baer and J. O'Farrill, JCAP 0404 (2004) 005; H. Baer, A. Belyaev, T. Krupovnickas and J. O'Farrill, Ref.11.

[29] HESS Collaboration, F. Aharonian et al. Astron. Astrophys. 425 (2004) L13; F. Aharonian et al. Science 307 (2005) 1938; F. Aharonian et al. Nature 439 (2006) 695.

[30] J. Albert et al. (MAGIC Collaboration), Astrophys. J. 638 (2006) L101; J. Albert et al. astro-ph/0604197, Ap. J. Lett. (in press).

[31] J.F. Navarro et al., Mon. Not. Roy. Astron. Soc. 349 (2004) 1039, astro-ph/0311231; the adiabatic contraction of the halo follows Blumental et al., Astrophys. J. 301 (1986) 27. For the halo parameter choices see also ref. [37.

[32] A. Burkert, Astrophys. J. 447 (1995) L25.

[33] S. Profumo and P. Ullio, JCAP 0407 (2004) 006, [arXiv:hep-ph/0406018].

[34] T. Sjöstrand, Comput. Phys. Commun. 82 (1994) 74; T. Sjöstrand, PYTHIA 5.7 and JETSET 7.4. Physics and Manual, CERN-TH.7112/93, arXiv:hep-ph/9508391 (revised version). 
[35] F. Donato, N. Fornengo and P. Salati, Phys. Rev. D62 (2000) 043003;

[36] L.J. Gleeson and W.I. Axford, Astrophys. J. 149 (1967) L115.

[37] S. Profumo and P. Ullio, JCAP 0407 (2004) 006.

[38] S. Profumo and C.E. Yaguna, Phys. Rev. D 70 (2004) 095004.

[39] K. Mori, C. J. Hailey, E. A. Baltz, W. W. Craig, M. Kamionkowski, W. T. Serber and P. Ullio, Astrophys. J. 566 (2002) 604; C. J. Hailey et al., JCAP 0601 (2006) 007.

[40] H. Baer and S. Profumo, JCAP 0512 (2005) 008.

[41] V. Berezinski et al. Astropart. Phys. 5 (1996) 1; R. Arnowitt and P. Nath, Phys. Rev. D 56 (1997) 2820; J. Ellis, K. Olive and Y. Santoso, Phys. Lett. B 539 (2002) 107; J. Ellis, T. Falk, K. Olive and Y. Santoso, Nucl. Phys. B 652 (2003) 259; M. Drees, hep-ph/0410113 (2004); H. Baer et al., Ref.[14].

[42] R. Arnowitt, B. Dutta, T. Kamon, N. Kolev and D. Toback, hep-ph/0603128 (2006).

[43] R. Kitano and Y. Nomura, Phys. Lett. B 632 (2006) 162, and hep-ph/0602096. 


\section{ZMW : $m_{3 / 2}=11.5 \mathrm{TeV}, \tan \beta=10, \mu>0, m_{t}=175 \mathrm{GeV}$}
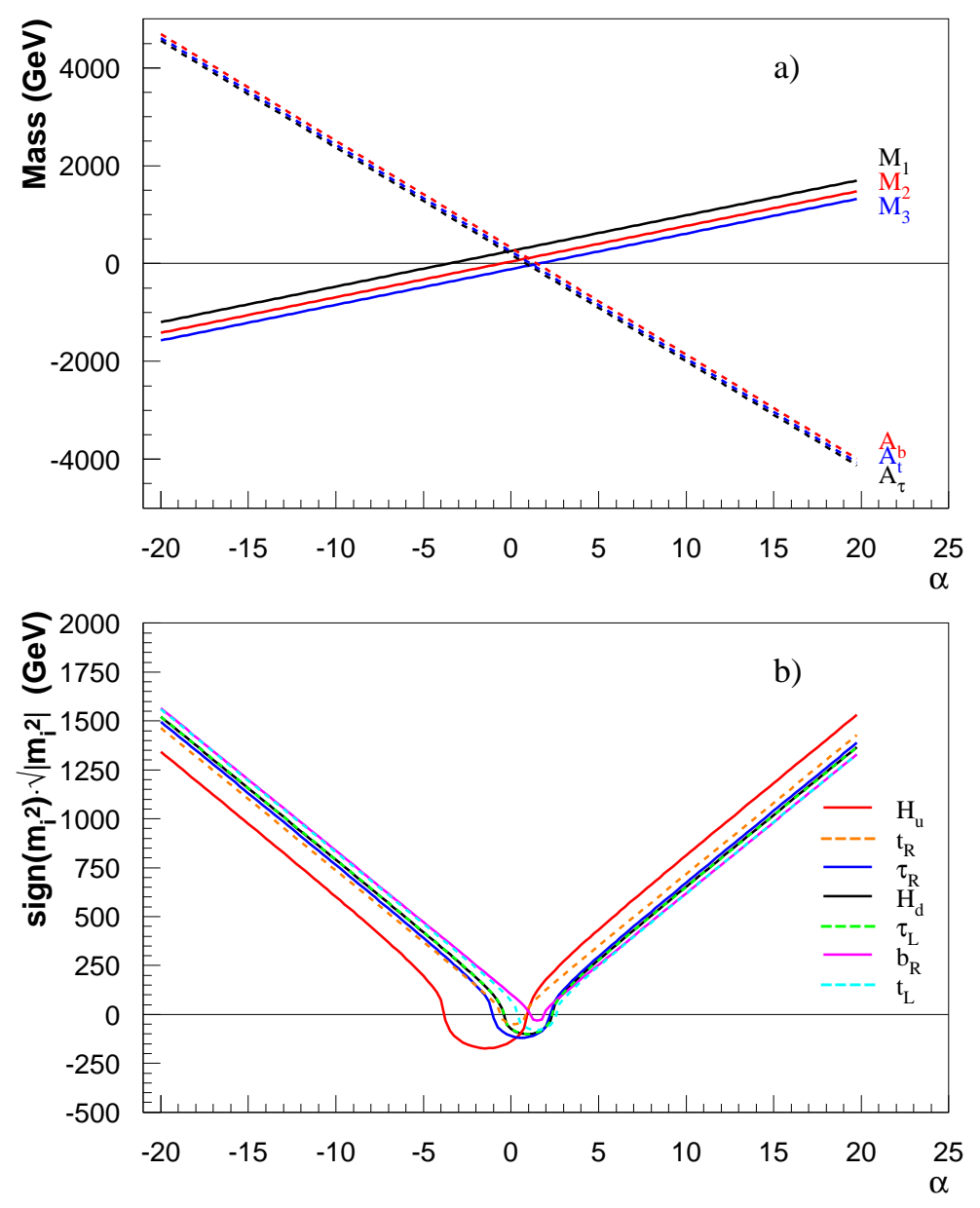

Figure 1: Various soft SUSY breaking parameters at the scale $Q=M_{G U T}$ versus $\alpha$ for $n_{i}=0$, $\ell_{a}=1, m_{3 / 2}=11.5 \mathrm{TeV}, \tan \beta=10, \mu>0$ and $m_{t}=175 \mathrm{GeV}$. In $a$ ), we show gaugino masses and $A$ terms, while in $b$ ) we show $\operatorname{sign}\left(m_{i}^{2}\right) \cdot \sqrt{\left|m_{i}^{2}\right|}$ for third generation scalar and Higgs boson soft SUSY breaking masses. 
ZMW: $\alpha=6, \mathrm{~m}_{3 / 2}=11.5 \mathrm{TeV}, \tan \beta=10, \mu>0, \mathrm{~m}_{\mathrm{t}}=175 \mathrm{GeV}$

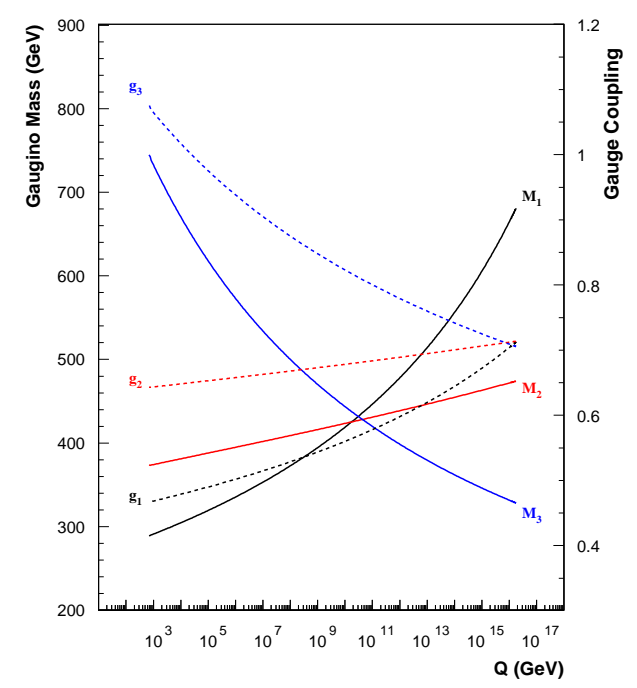

ZMW: $\alpha=6, m_{3 / 2}=11.5 \mathrm{TeV}, \tan \beta=10, \mu>0, m_{t}=175 \mathrm{GeV}$

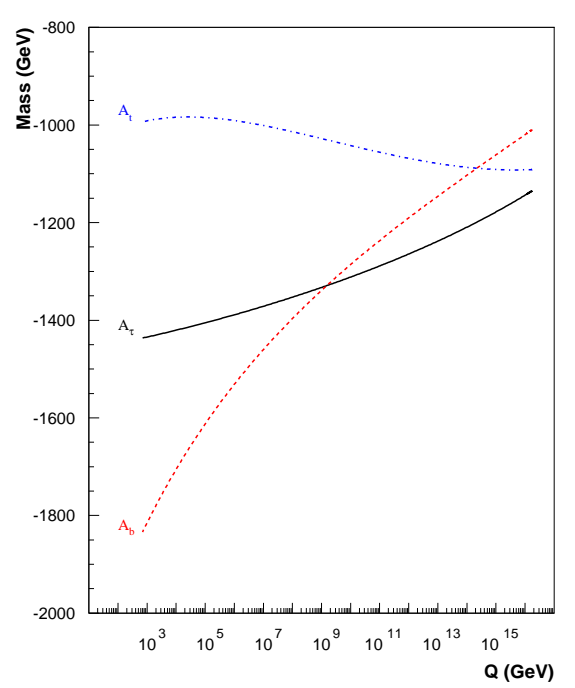

Figure 2: Evolution of $a$ ) the gaugino masses $M_{1}, M_{2}$ and $M_{3}$, and of $b$ ), the trilinear soft masses $A_{t}, A_{b}$ and $A_{\tau}$, from $Q=M_{G U T}$ to $Q=M_{\text {weak }}$ for the model with zero modular weights with $\alpha=6, m_{3 / 2}=11.5 \mathrm{TeV}, \tan \beta=10, \mu>0$ and $m_{t}=175 \mathrm{GeV}$. Also shown (right hand scale) is the corresponding evolution of the three gauge couplings. 
$m_{3 / 2}=11.5 \mathrm{TeV}, \tan \beta=10, \mu>0, m_{t}=175 \mathrm{GeV}$

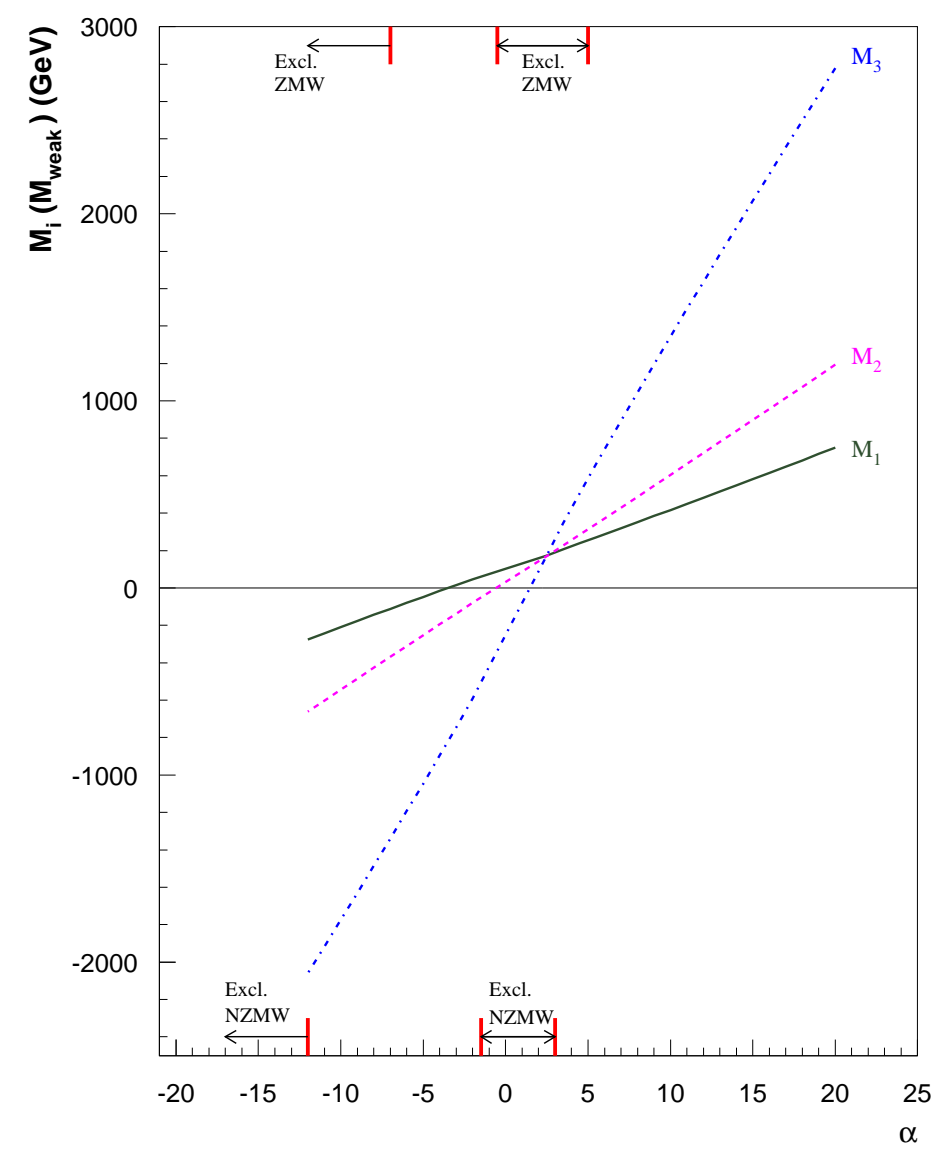

Figure 3: SSB gaugino mass parameters at the weak scale versus the MM-AMSB model $\alpha$ for $m_{3 / 2}=11.5 \mathrm{TeV}, \tan \beta=10, \mu>0$. For a value of $\alpha$ close to the mirage unification point, mixed wino dark matter may be possible, while for $\alpha \sim-1.75$, bino-wino coannihilation could lead to an acceptable dark matter relic density provided these values of $\alpha$ are allowed by other constraints. The ranges of $\alpha$ excluded for the model with zero modular weights (ZMW) for matter fields are shown on the top, while the corresponding excluded ranges for the particular choice of non-zero modular weights (NZMW) $n_{H_{u}}=n_{H_{d}}=1, m_{\text {matter }}=1 / 2$, is shown at the bottom. 
ZMW: $\alpha=6, m_{3 / 2}=11.5 \mathrm{TeV}, \tan \beta=10, \mu>0, \mathrm{~m}_{\mathrm{t}}=175 \mathrm{GeV}$

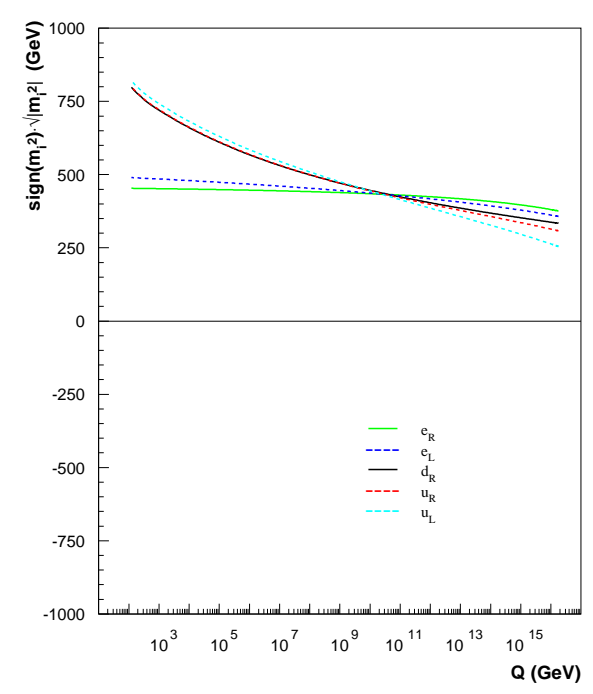

ZMW: $\alpha=6, m_{3 / 2}=11.5 \mathrm{TeV}, \tan \beta=10, \mu>0, m_{t}=175 \mathrm{GeV}$

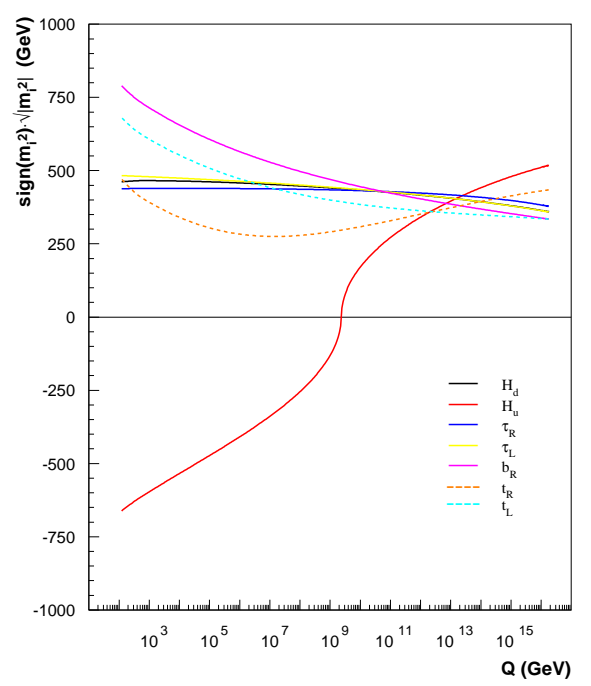

Figure 4: Evolution of scalar soft masses $m_{i}^{2}$ for $a$ ) first generation scalars and $b$ ) third generation and Higgs scalars from $Q=M_{G U T}$ to $Q=M_{\text {weak }}$ for $\alpha=6, m_{3 / 2}=11.5 \mathrm{TeV}, \tan \beta=10$ and $\mu>0$, with $\ell_{a}=1$ and modular weights set to zero. To show evolution to negative squared masses, we actually plot $\operatorname{sign}\left(m_{i}^{2}\right) \cdot \sqrt{\left|m_{i}^{2}\right|}$.

\section{ZMW : $m_{3 / 2}=11.5 \mathrm{TeV}, \mathrm{m}_{\mathrm{t}}=175 \mathrm{GeV}$}

a) $\tan \beta=10, \mu>0$

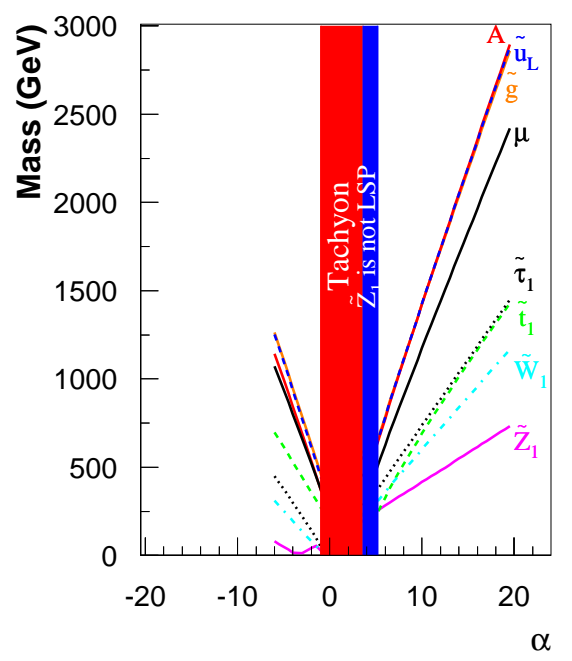

b) $\tan \beta=30, \mu>0$

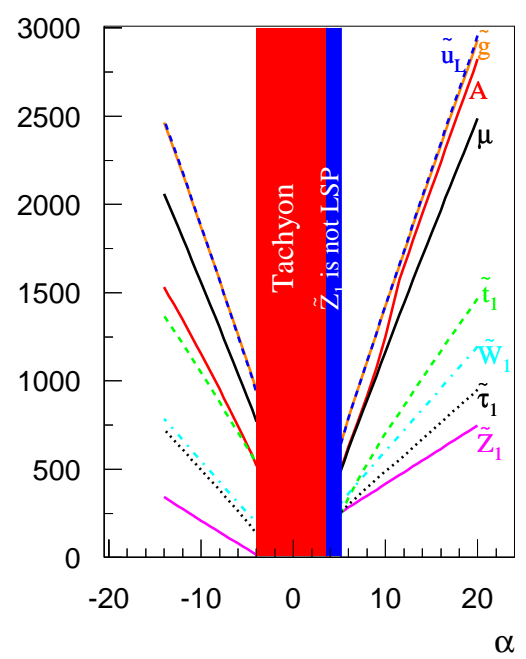

Figure 5: Sparticle masses vs. $\alpha$ in the MM-AMSB model with zero modular weights, for $m_{3 / 2}=$ $11.5 \mathrm{TeV}$ and $\mu>0$. We plot for $a) \tan \beta=10$ and $b) \tan \beta=30$. 


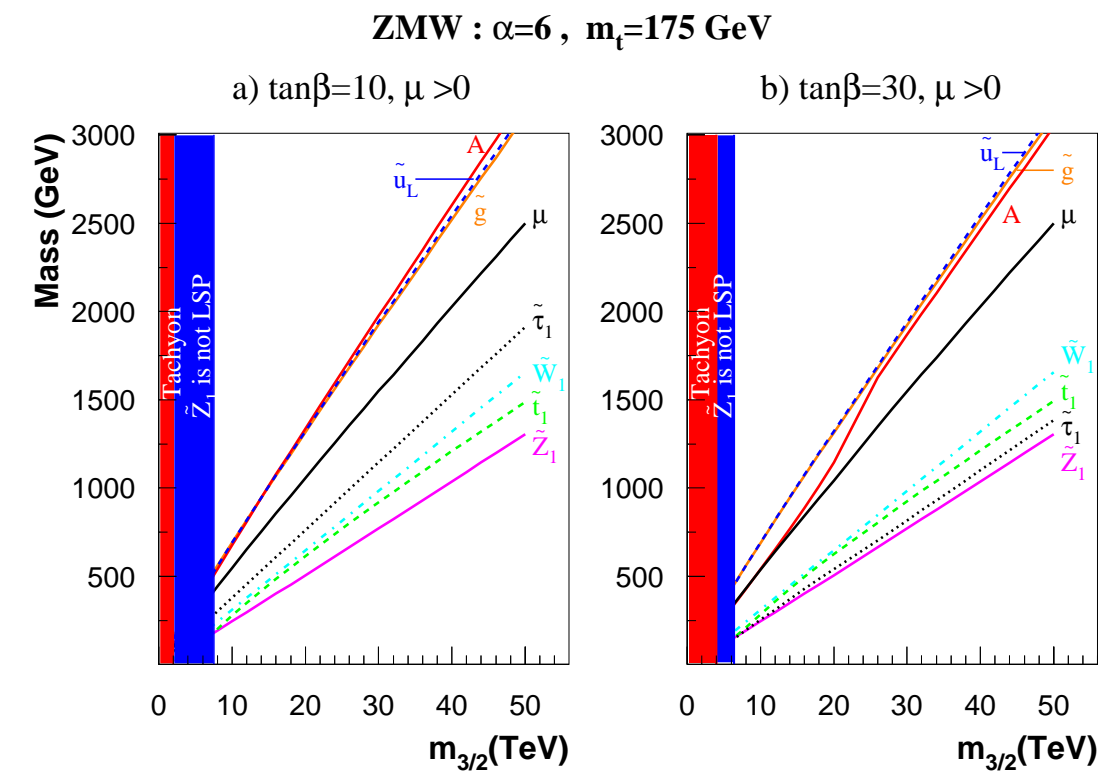

Figure 6: Sparticle masses vs. $m_{3 / 2}$ in the MM-AMSB model with zero modular weights, for $\alpha=6$ and $\mu>0$. We plot for $a) \tan \beta=10$ and $b) \tan \beta=30$.

ZMW : $\alpha=6, m_{3 / 2}=11.5 \mathrm{TeV}, m_{t}=175 \mathrm{GeV}$

a) $\mu>0$

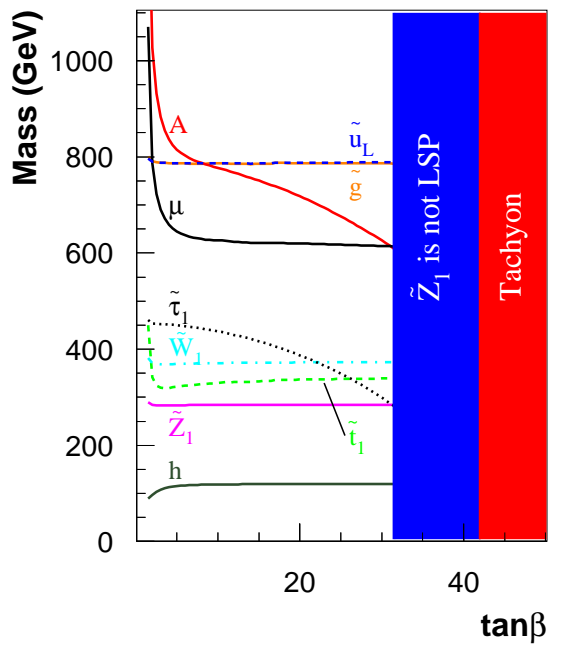

b) $\mu<0$

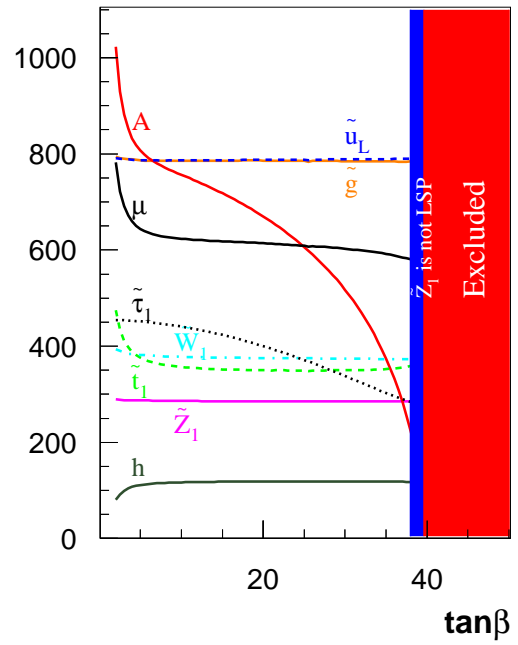

Figure 7: Sparticle masses vs. $\tan \beta$ in the MM-AMSB model with zero modular weights, for $\alpha=6$ and $m_{3 / 2}=11.5 \mathrm{TeV}$, with $\left.a\right) \mu>0$ and $\left.b\right) \mu<0$. 
ZMW : $\alpha=6, m_{3 / 2}=11.5 \mathrm{TeV}, m_{\mathrm{t}}=175 \mathrm{GeV}$

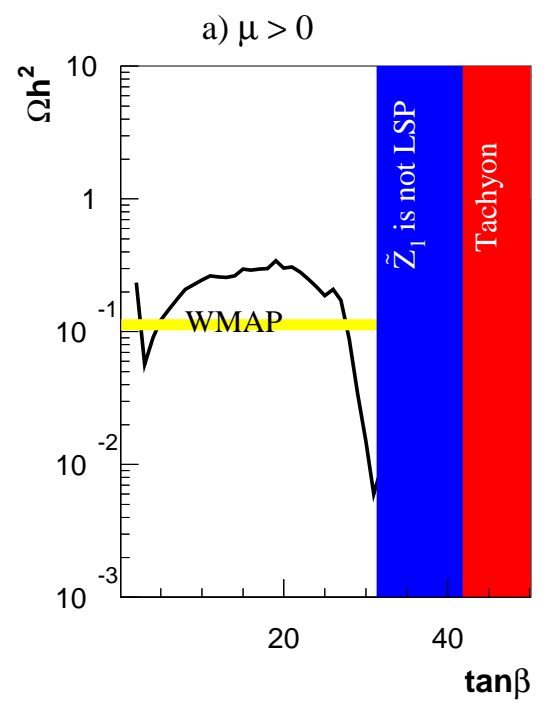

b) $\mu<0$

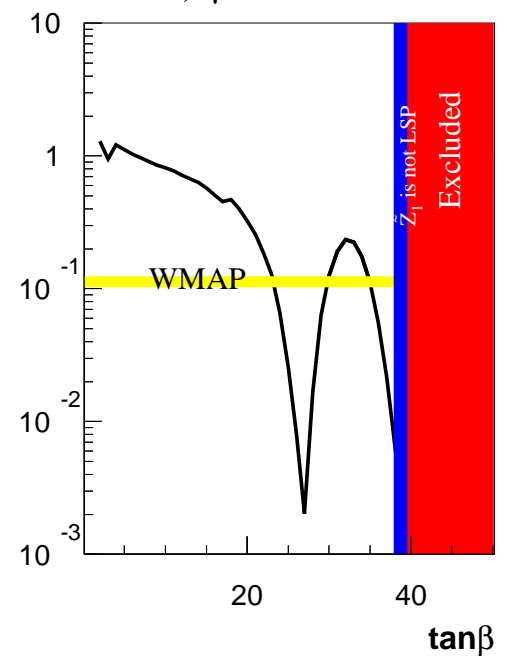

Figure 8: The neutralino relic density $\Omega_{\widetilde{Z}_{1}} h^{2}$ vs. $\tan \beta$ for the MM-AMSB model with zero modular weights, for $\alpha=6$ and $m_{3 / 2}=11.5 \mathrm{TeV}$, with $\left.a\right) \mu>0$ and $\left.b\right) \mu<0$. 
Gravitino mass vs. $\alpha, \tan \beta=10, \mu>0$, ZMW

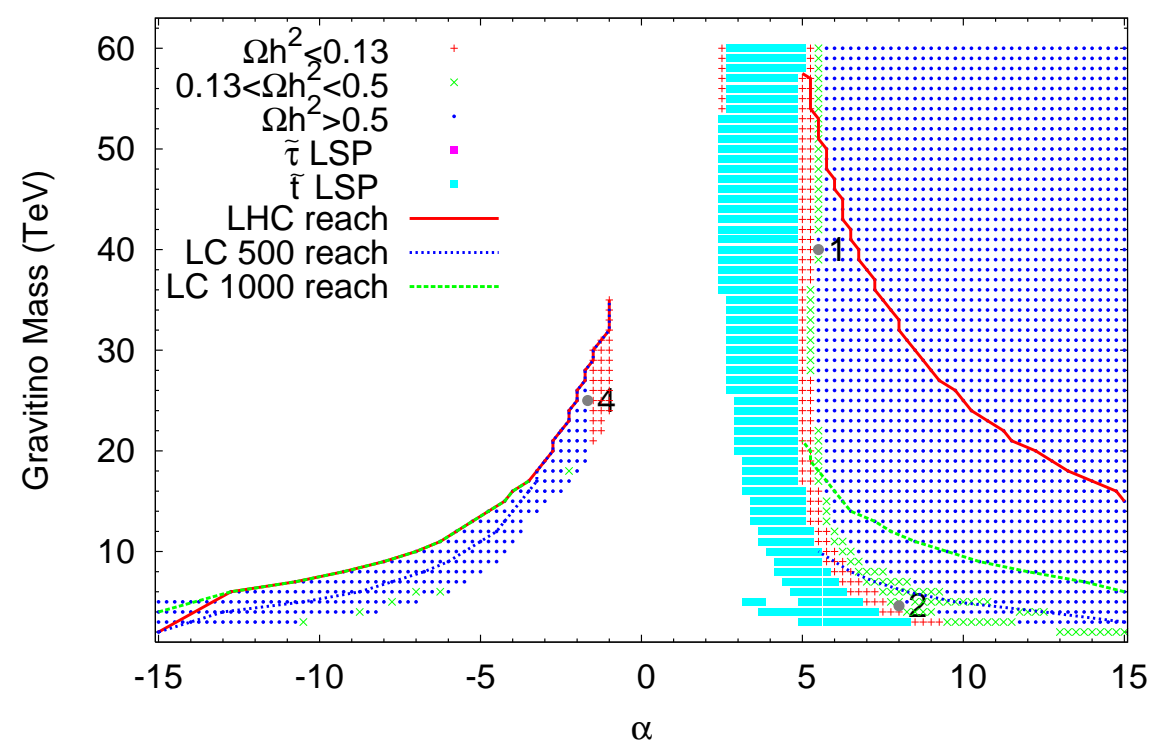

Gravitino mass vs. $\alpha, \tan \beta=30, \mu>0, \mathrm{ZMW}$

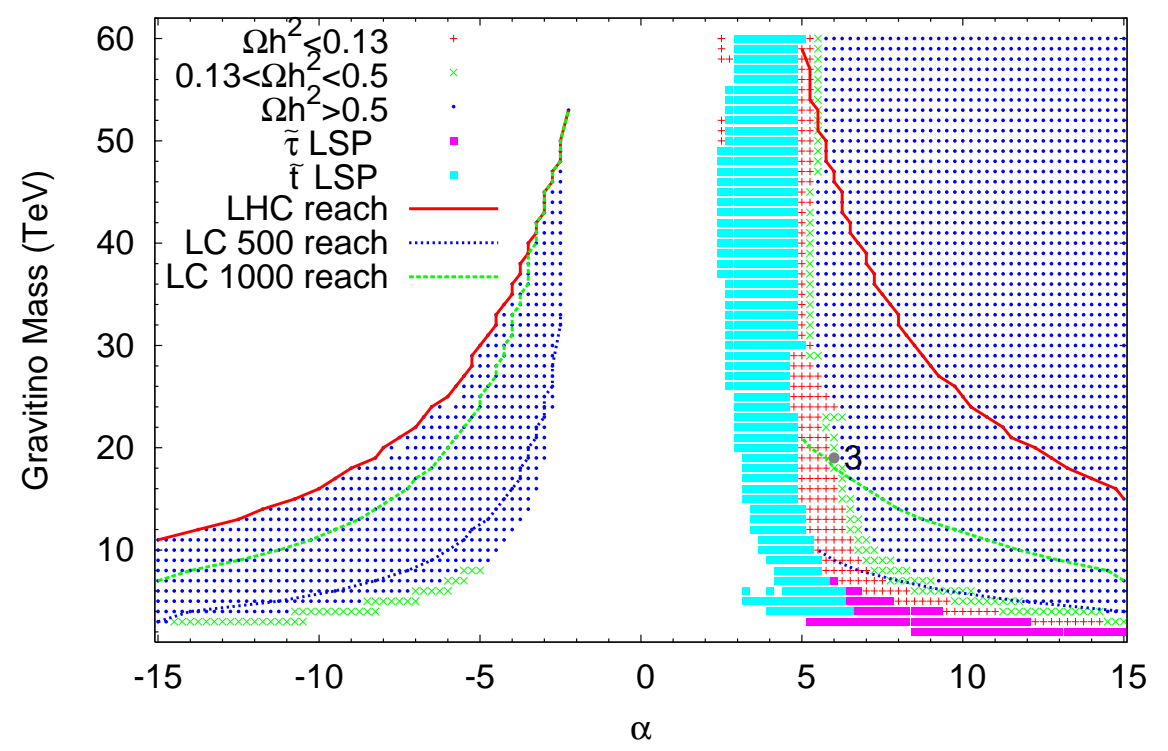

Figure 9: Regions of the $\alpha$ vs. $m_{3 / 2}$ plane of the MM-AMSB model where electroweak symmetry is radiatively broken to electromagnetism and which are consistent with experimental constraints from LEP2 searches for $a) \tan \beta=10, \mu>0$ and $b) \tan \beta=30, \mu>0$. Turquoise (magenta) squares denote points where the $t$-squark (tau slepton) is the LSP. The ranges of relic density are shown on the figure. We also show the approximate reach of the CERN LHC for $100 \mathrm{fb}^{-1}$ of integrated luminosity, and the kinematic reach of a $\sqrt{s}=0.5$ and $1 \mathrm{TeV}$ linear $e^{+} e^{-}$collider. 
NZMW : $m_{3 / 2}=11.5 \mathrm{TeV}, \tan \beta=10, \mu>0, m_{t}=175 \mathrm{GeV}$
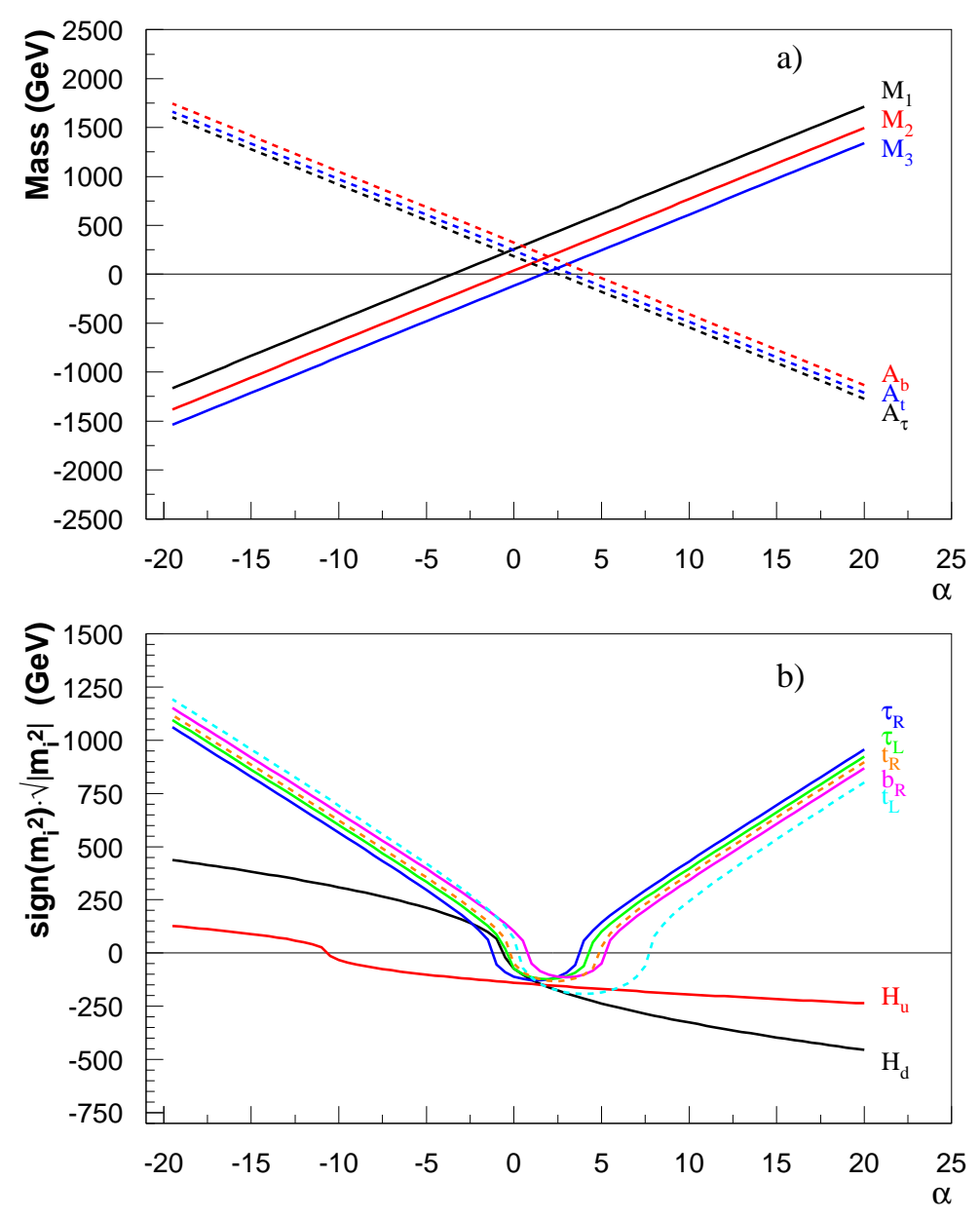

Figure 10: Various soft SUSY breaking parameters at the scale $Q=M_{G U T}$ versus $\alpha$ for the NZMW model with $n_{\text {matter }}=\frac{1}{2}, n_{\text {Higgs }}=1, \ell_{a}=1, m_{3 / 2}=11.5 \mathrm{TeV}, \tan \beta=10, \mu>0$ and $m_{t}=175 \mathrm{GeV}$. In $a$ ), we show gaugino masses and $A$ terms, while in $b$ ) we show $\operatorname{sign}\left(m_{i}^{2}\right) \cdot \sqrt{\left|m_{i}^{2}\right|}$ for third generation and Higgs soft breaking masses. 
NZMW: $\alpha=6, \mathrm{~m}_{3 / 2}=11.5 \mathrm{TeV}, \tan \beta=10, \mu>0, \mathrm{~m}_{\mathrm{t}}=175 \mathrm{GeV}$

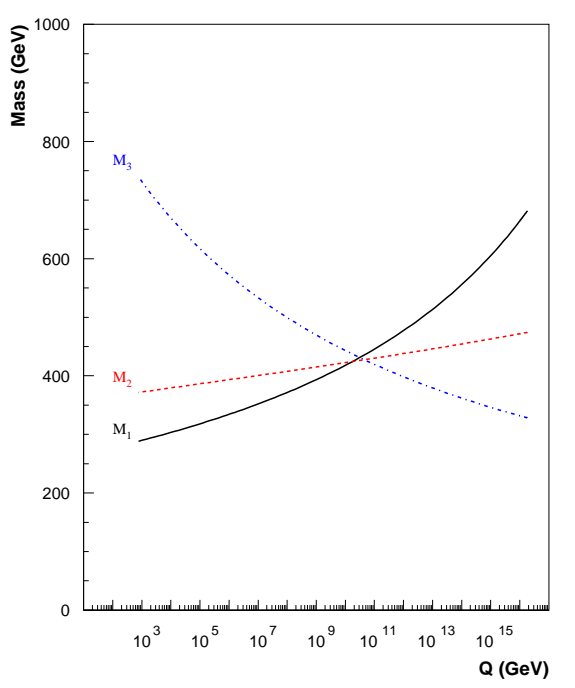

NZMW: $\alpha=6, m_{3 / 2}=11.5 \mathrm{TeV}, \tan \beta=10, \mu>0, m_{\mathrm{t}}=175 \mathrm{GeV}$

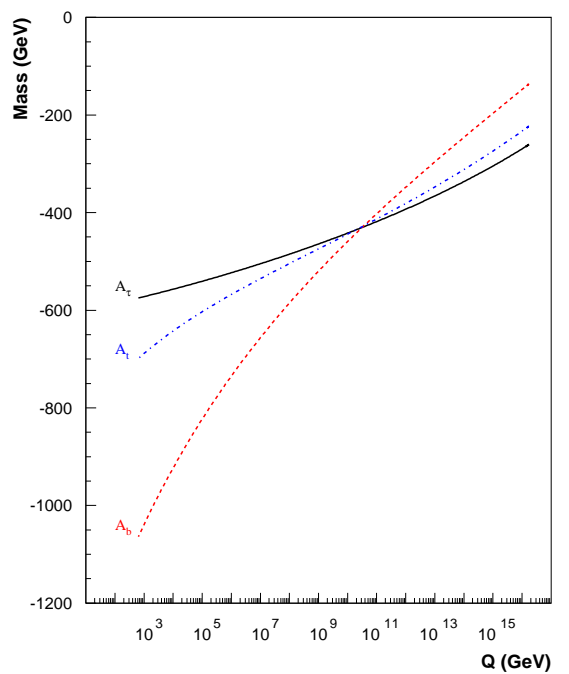

Figure 11: Evolution of $a$ ) the gaugino masses $M_{1}, M_{2}$ and $M_{3}$ from $Q=M_{G U T}$ to $Q=M_{\text {weak }}$ in the NZMW model for $\alpha=6, m_{3 / 2}=11.5 \mathrm{TeV}, \tan \beta=10, \mu>0$ and $m_{t}=175 \mathrm{GeV}$. In frame $b$, we show evolution of the $A_{i}$ trilinear soft masses $A_{t}, A_{b}$ and $A_{\tau}$ from $Q=M_{G U T}$ to $Q=M_{\text {weak }}$ for the same parameter choices. 


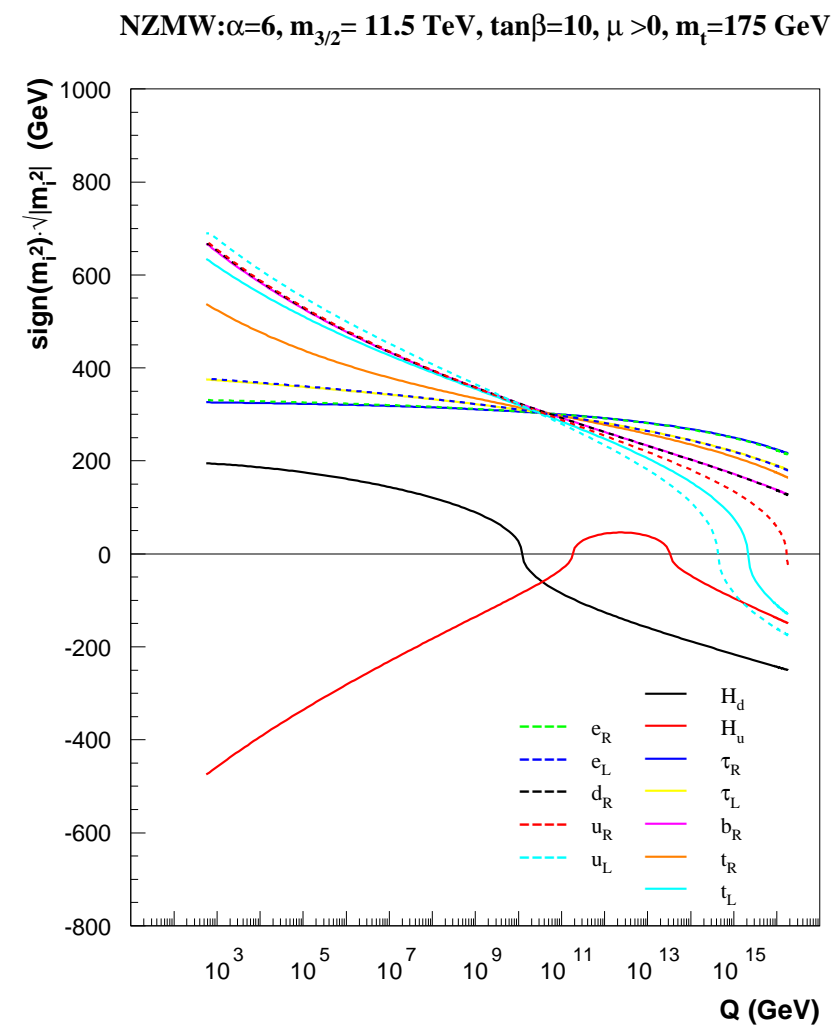

Figure 12: Evolution of scalar soft masses $m_{i}^{2}$ for $a$ ) first generation scalars and $b$ ) third generation and Higgs scalars from $Q=M_{G U T}$ to $Q=M_{\text {weak }}$ for $\alpha=6, m_{3 / 2}=11.5 \mathrm{TeV}, \tan \beta=10$ and $\mu>0$ for the case of non-zero modular weights. We actually plot $\operatorname{sign}\left(m_{i}^{2}\right) \cdot \sqrt{\left|m_{i}^{2}\right|}$. 
NZMW : $m_{3 / 2}=11.5 \mathrm{TeV}, m_{t}=175 \mathrm{GeV}$
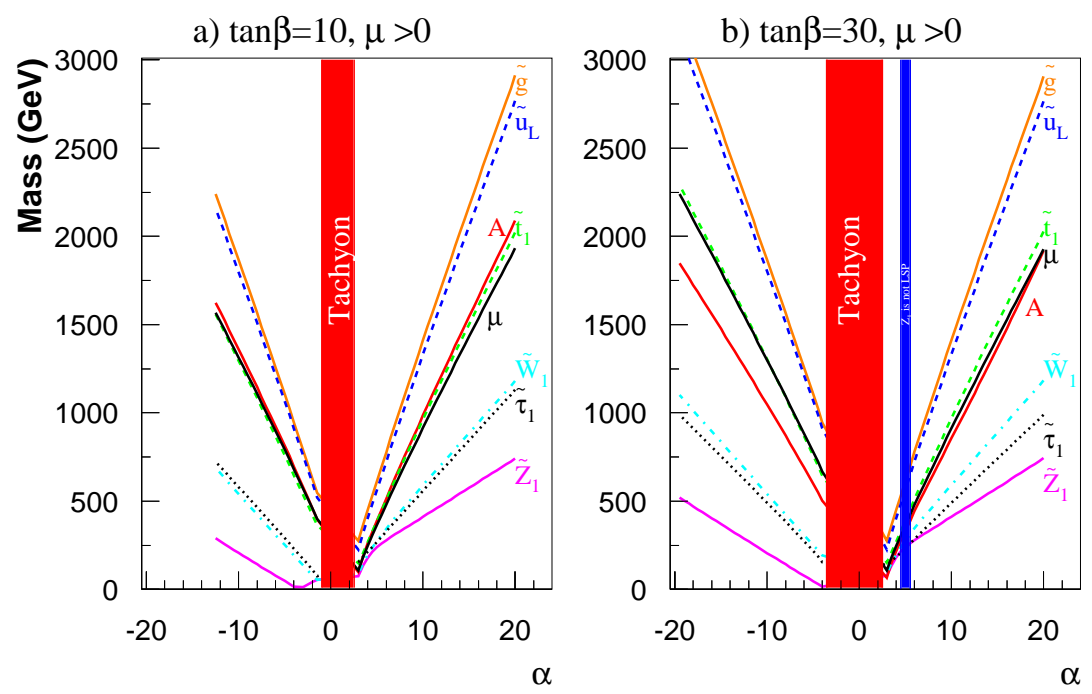

Figure 13: Sparticle masses vs. $\alpha$ in the NZMW model, for $m_{3 / 2}=11.5 \mathrm{TeV}$ and $\mu>0$. We plot for $a) \tan \beta=10$ and $b) \tan \beta=30$.

NZMW : $\alpha=6, m_{3 / 2}=11.5 \mathrm{TeV}, m_{t}=175 \mathrm{GeV}$

a) $\mu>0$

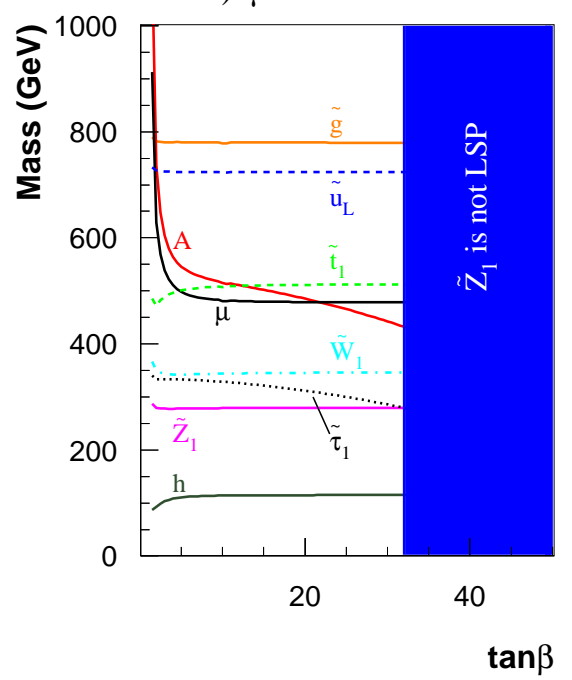

b) $\mu<0$

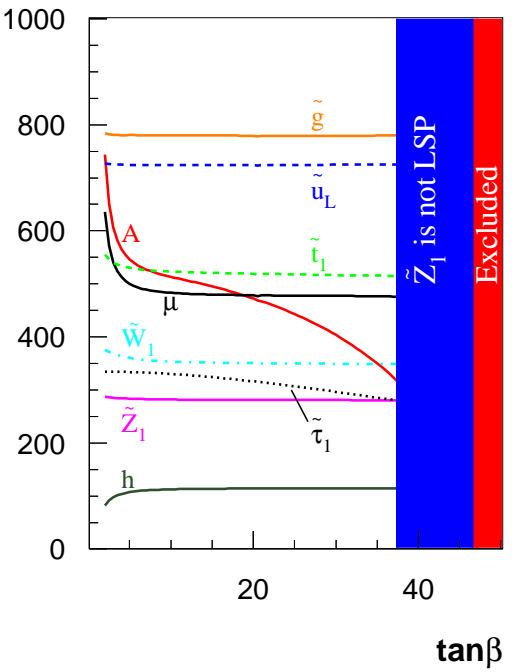

Figure 14: Sparticle masses vs. $\tan \beta$ in the NZMW model for $\alpha=6$ and $m_{3 / 2}=11.5$. We plot for $a) \mu>0$ and $b) \mu<0$. 
NZMW : $\alpha=6, m_{3 / 2}=11.5 \mathrm{TeV}, m_{t}=175 \mathrm{GeV}$

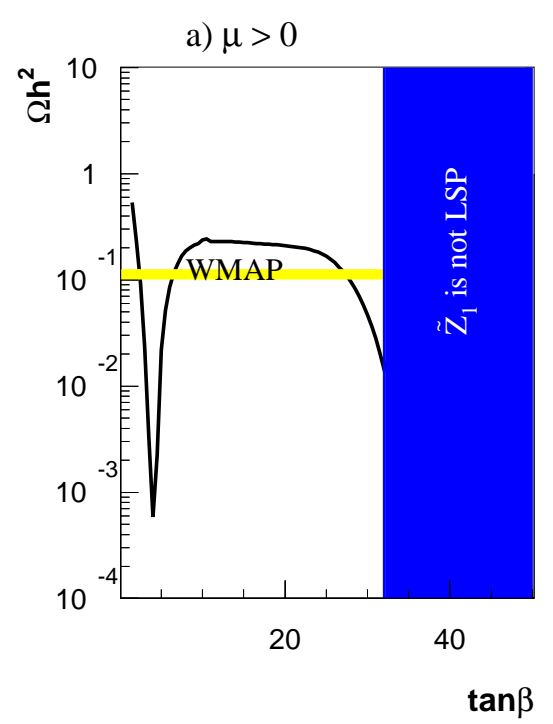

b) $\mu<0$

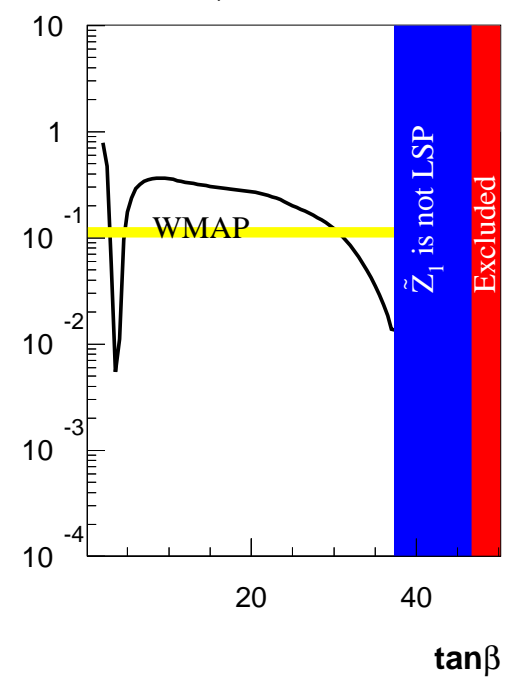

Figure 15: The relic density $\Omega_{\widetilde{Z}_{1}} h^{2}$ vs. $\tan \beta$ for the NZMW model with $\alpha=6$ and $m_{3 / 2}=11.5$. We plot for $a) \mu>0$ and $b) \mu<0$. 
Gravitino mass vs. $\alpha, \tan \beta=10, \mu>0, N Z M W$

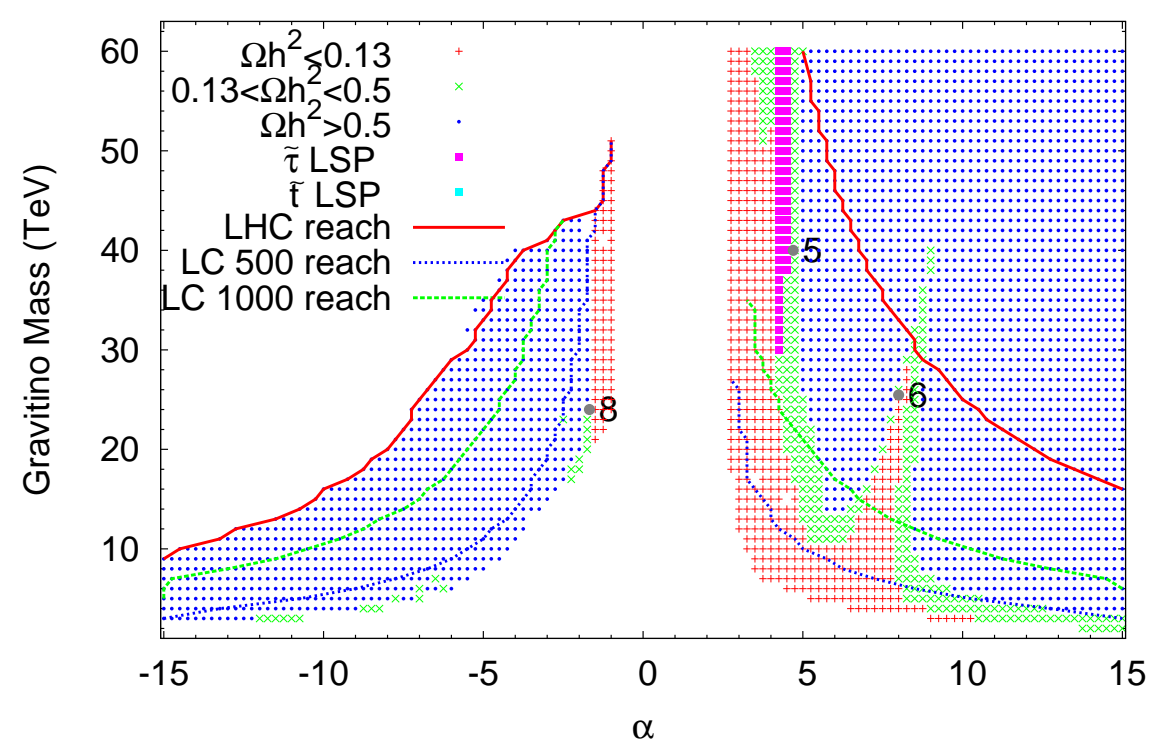

Gravitino mass vs. $\alpha, \tan \beta=30, \mu>0, N Z M W$

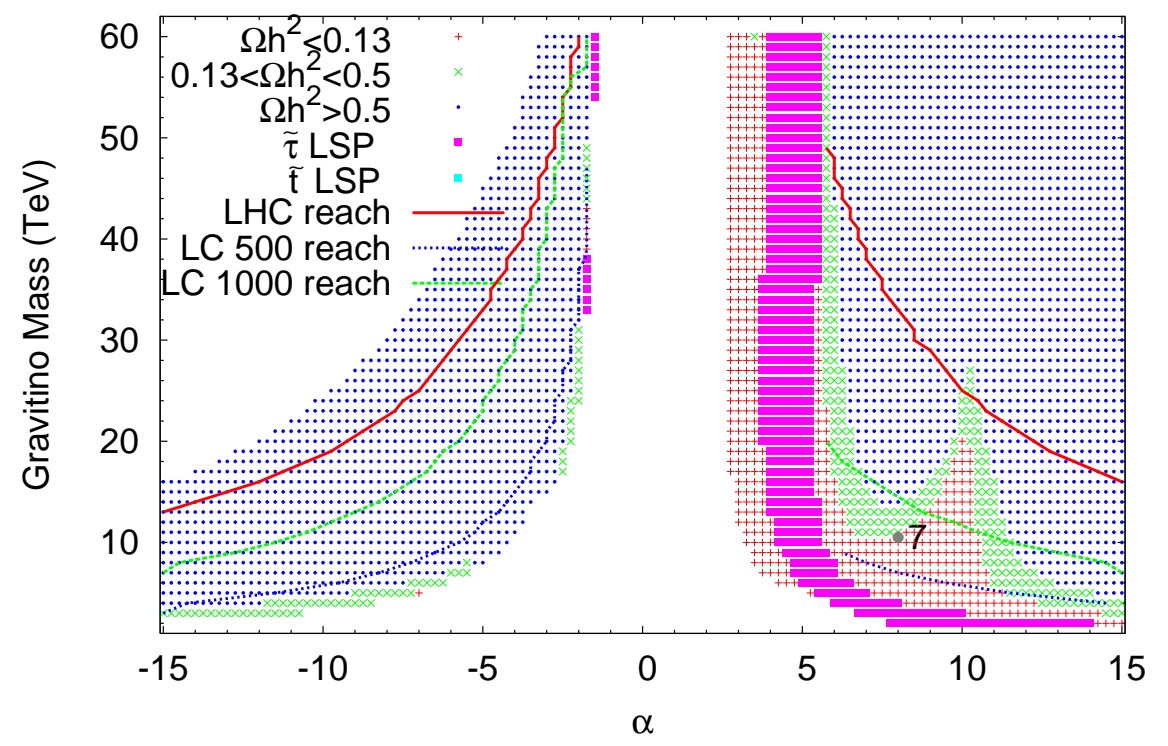

Figure 16: Regions of the $\alpha$ vs. $m_{3 / 2}$ plane allowed by theory constraints, LEP2 searches and neutralino relic density for $a) \tan \beta=10, \mu>0$ and $b) \tan \beta=30, \mu>0$, and non-zero modular weights. We also show the approximate reach of the CERN LHC for $100 \mathrm{fb}^{-1}$ of integrated luminosity, along with the kinematic reach of a $\sqrt{s}=0.5$ and of a $1 \mathrm{TeV}$ linear $e^{+} e^{-}$collider. 


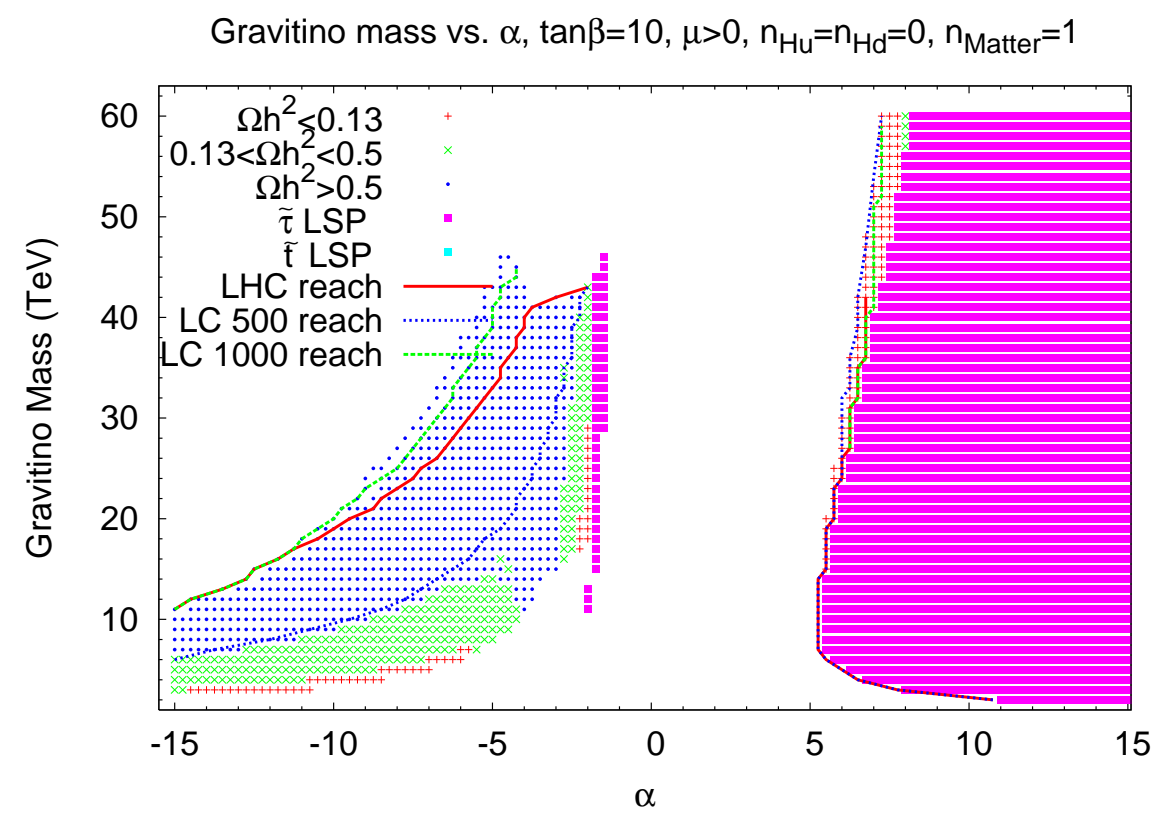

Figure 17: Regions of the $\alpha$ vs. $m_{3 / 2}$ plane allowed by theory constraints, LEP2 searches and neutralino relic density for $\tan \beta=10, \mu>0$ and the choice $n_{H_{u}}=n_{H_{d}}=0$ and $n_{\text {matter }}=1$ for the modular weights. We also show the approximate reach of the CERN LHC for $100 \mathrm{fb}^{-1}$ of integrated luminosity, along with the kinematic reach of a $\sqrt{s}=0.5$ and $1 \mathrm{TeV}$ linear $e^{+} e^{-}$collider. 